\title{
Scale decomposition in compressible turbulence
}

\author{
Hussein Aluie \\ Applied Mathematics and Plasma Physics (T-5), \\ Computer, Computational, and Statistical Sciences (CCS-2), \\ 83 Center for Non-linear Studies, \\ Los Alamos National Laboratory, MS-B258 Los Alamos, NM 87545, USA
}

\begin{abstract}
This work presents a rigorous framework based on coarse-graining to analyze highly compressible turbulence. We show how the requirement that viscous effects on the dynamics of large-scale momentum and kinetic energy be negligible - an inviscid criterion - naturally supports a density weighted coarse-graining of the velocity field. Such a coarse-graining method is already known in the literature as Favre filtering; however its use has been primarily motivated by appealing modeling properties rather than underlying physical considerations. We also prove that kinetic energy injection can be localized to the largest scales by proper stirring, and argue that stirring with an external acceleration field rather than a body force would yield a longer inertial range in simulations. We then discuss the special case of buoyancy-driven flows subject to a spatially-uniform gravitational field. We conclude that a range of scales can exist over which the mean kinetic energy budget is dominated by inertial processes and is immune from contributions due to molecular viscosity and external stirring.
\end{abstract}

Key Words: Compressible turbulence, Scale decomposition, Inertial range, Favre filtering 


\section{Introduction}

The aim of this paper is to give a systematic, theoretical approach based on coarse-graining (or filtering) to analyze non-linear scale interactions in compressible turbulent flows. It builds upon previous work of Germano Germano (1992) and Eyink Eyink (1995, 2005). There are several motivations for this work. First, there is no unique way to specifying a notion of scale, such as defining large-scale momentum and large-scale kinetic energy, in compressible turbulence. The traditional approach in this subject has employed density-weighted averaging, also known as Favre averaging, to decompose a flow into large-scale and turbulent components Favre (1969). Using Favre averaging, density, $\rho(\mathbf{x})$, and velocity, $\mathbf{u}(\mathbf{x})$, are combined to yield a large-scale momentum, $\langle\rho \mathbf{u}\rangle$, and a large-scale kinetic energy, $\frac{1}{2}\langle\rho \mathbf{u}\rangle^{2} /\langle\rho\rangle$, where $\langle\ldots\rangle$ can denote an ensemble-average, a time-average, or, as used in this paper, a space-average $\frac{1}{V} \int_{V} d \mathbf{x}(\ldots)$. However, such a decomposition has been primarily borne out of convenience to modelers and practitioners rather than physical considerations. It seems that a priori there is no fundamental reason to favor Favre averaging over any other combination of $\rho$ and $\mathbf{u}$, from an infinite number of possibilities, such as defining large-scale kinetic energy as $\left\langle\rho^{\alpha} \mathbf{u}\right\rangle \cdot\left\langle\rho^{1-\alpha} \mathbf{u}\right\rangle / 2$ for $0<\alpha<1$ Kida and Orszag (1990) or, alternatively, as $\langle\rho\rangle|\langle\mathbf{u}\rangle|^{2} / 2$ Chassaing (1985).

Second, and more importantly, this paper provides the foundation for recent work Aluie (2011); Aluie et al. (2012) in which we addressed basic questions about the nature of the cascade in compressible turbulence. While the classical ideas of Richardson, Kolmogorov, and Onsager form the cornerstone for our modern understanding of incompressible turbulence, there had been no grounds for extending such a theory to compressible flows. The potent ideas of an inertial range and universality are often invoked without physical basis in compressible turbulence. Elementary questions on the possible existence of a scale-range which is immune from direct effects of viscosity and large scale forcing, on whether energy is transferred to small scales through a cascade process, and whether such a cascade is local in scale, had not been previously addressed. Resolving these questions is necessary to warrant the concept of an inertial range and to justify the existence of universal statistics of turbulent fluctuations. Furthermore, Kolmogorov's 4/5-th law for the energy flux is an exact result which has no counterpart in compressible turbulence. An analogous result would be essential for attempting to predict the scaling of spectra and structure functions. Recent progress in this regard has been encouraging; Falkovich et al. Falkovich et al. (2010) derived a relation for compressible turbulence analogous to the Karman-Howarth relation

for incompressible turbulence, and Galtier and Banerjee Galtier and Banerjee (2011) derived an exact relation for the special case of compressible turbulence with an isothermal equation of state.

This work is also very useful from the standpoint of numerical modeling. Compressible flows, especially in astrophysical systems, often involve a huge range of scales which cannot be simulated directly. The coarse-graining approach provides a theoretical basis for constructing models of turbulence that 
may faithfully reflect the dynamics at unresolved scales. The formalism that we employ is the same as that used in large-eddy simulation (LES) modeling of turbulent flows. This work thus provides a theoretical complement to those modeling efforts. However, while the equations we analyze coincide (to a considerable extent) with those that are employed in LES of compressible turbulence, their use here will be for rather different purposes. In LES, plausible but uncontrolled closures are adopted for the subscale terms, whereas the aim here, as in Eyink (1995, 2005); Aluie (2011); Aluie et al. (2012), is to develop several exact estimates and some general physical understanding of these terms. Another difference is that LES generally takes the scale parameter $\ell$ to be a fixed length of the order of the "integral scale" $L$. Our interest here is rather to probe all scales in the flow, including limits of small $\ell \ll L$.

We shall show below how a decomposition based on Favre filtering (and averaging) comes out naturally from the physical requirement that viscous effects have a negligible role in the dynamics of large-scale momentum and large-scale kinetic energy. We call this the inviscid criterion. Using the coarse-graining approach, we will prove rigorously the existence of an intermediate range of scales over which viscous dissipation and external kinetic energy injection can be made to vanish. Our decomposition also leads to two terms responsible for transferring kinetic energy across scales and constitute the so-called "subgrid scale flux," which we shall discuss in detail.

This paper is intended for an audience with diverse backgrounds. For that reason, we have attempted to present the underlying physical ideas at an intuitive level in the main text while leaving mathematical details and rigorous proofs to appendices. The outline is as follows. In Section 2 we present preliminary definitions and discussion. In Section 3 we show how viscous dynamics can be isolated to the smallest scales by a proper scale-decomposition and in Section 4 we discuss the inertial dynamics based on such a decomposition. In Section 5 we prove that it is possible to localize kinetic energy injection to the largest scales in a system and discuss buoyancy-driven (Rayleigh-Taylor) flows as a special case. In Section 6. we examine contributions from compressibility effects to the flux of kinetic energy across scales. We conclude with Section 7 and three appendices which contain detailed proofs of our results.

\section{Preliminaries}

\subsection{Governing dynamics}

In this paper we study the dynamics at various scales through a direct analysis of the compressible Navier Stokes equations without the use of any closure approximation. The equations are those of continuity 
(1), momentum (2), and either internal energy (3) or total energy (4):

$$
\begin{array}{cl}
\partial_{t} \rho & +\partial_{j}\left(\rho u_{j}\right)=0 \\
\partial_{t}\left(\rho u_{i}\right) & +\partial_{j}\left(\rho u_{i} u_{j}\right)=-\partial_{i} P+\partial_{j} \sigma_{i j}+\rho F_{i} \\
\partial_{t}(\rho e) & +\partial_{j}\left\{\rho e u_{j}\right\}=-P \partial_{j} u_{j}+2 \mu\left(\left|S_{i j}\right|^{2}-\frac{1}{d}\left|S_{k k}\right|^{2}\right)-\partial_{j} q_{j} \\
\partial_{t}(\rho E) & +\partial_{j}\left(\rho E u_{j}\right)=-\partial_{j}\left(P u_{j}\right)+\partial_{j}\left[2 \mu u_{i}\left(S_{i j}-\frac{1}{d} S_{k k} \delta_{i j}\right)\right]-\partial_{j} q_{j}+\rho u_{i} F_{i} \\
\partial_{t}\left(\rho \frac{|\mathbf{u}|^{2}}{2}\right)+\partial_{j}\left\{\left(\rho \frac{|\mathbf{u}|^{2}}{2}+P\right) u_{j}-2 \mu\left(u_{i} S_{i j}-\frac{1}{d} u_{j} S_{k k}\right)\right\}=P \partial_{j} u_{j}-2 \mu\left(\left|S_{i j}\right|^{2}-\frac{1}{d}\left|S_{k k}\right|^{2}\right)+\rho u_{i} F_{i} .
\end{array}
$$

We also write down the kinetic energy budget (5) for convenience. Here, $\mathbf{u}$ is velocity, $\rho$ is density, $e$ is internal energy per unit mass, $E=|\mathbf{u}|^{2} / 2+e$ is total energy per unit mass, $P$ is pressure, $\mu$ is dynamic viscosity, $\mathbf{F}$ is an external acceleration field stirring the fluid, $\mathbf{q}=-\kappa \boldsymbol{\nabla} T$ is the heat flux with a conduction coefficient $\kappa$ and temperature $T$. The symmetric strain tensor is $S_{i j}=\left(\partial_{j} u_{i}+\partial_{i} u_{j}\right) / 2$, and the viscous stress, $\sigma_{i j}=2 \mu\left(S_{i j}-d^{-1} S_{k k} \delta_{i j}\right)$, is deviatoric (traceless) in $d$-dimensions. For convenience, we have assumed a zero bulk viscosity even though all our analysis applies to the more general case. Dynamic viscosity varies in space and is well-described by Sutherland's law or simpler power law approximations, $\mu(\mathbf{x}) \sim T^{\alpha}(\mathbf{x})$.

\subsection{Coarse-graining}

We first present a general approach to analyzing scale interactions in a turbulent flow. Following Leonard Leonard (1974), Germano Germano (1992), and Eyink Eyink (2005), we use a simple filtering technique common in the LES literature to resolve turbulent fields simultaneously in scale and in space.

For any field $\mathbf{a}(\mathbf{x})$, a "coarse-grained" or (low-pass) filtered field, which contains modes at scales $>\ell$, is defined in $d$-dimensions as

$$
\overline{\mathbf{a}}_{\ell}(\mathbf{x})=\int d^{d} \mathbf{r} G_{\ell}(\mathbf{r}) \mathbf{a}(\mathbf{x}+\mathbf{r}),
$$

where $G(\mathbf{r})$ is a convolution kernel. It can be any real-valued function which is sufficiently smooth, decays sufficiently rapidly for large $r$, and is normalized so that $\int d^{d} \mathbf{s} G(\mathbf{s})=1$ for dimensionless $\mathbf{s}$. It is assumed furthermore that $G$ is centered, $\int d^{d} \mathbf{s} \mathbf{s} G(\mathbf{s})=\mathbf{0}$, and with the main support in a ball of unit radius, $\int d^{d} \mathbf{s}|\mathbf{s}|^{2} G(\mathbf{s})=\mathcal{O}(1)$. Its dilation in a $d$-dimensional domain $\Omega, G_{\ell}(\mathbf{r}) \equiv \ell^{-d} G(\mathbf{r} / \ell)$, will share these properties except that its main support will be in a ball of radius $\ell$. If $G(\mathbf{s})$ is also non-negative, then (6) may be interpreted as a local space average. Note that $G(\mathbf{s})$ can be chosen so that both it and its Fourier transform $\hat{G}(\mathbf{k})$ are positive and infinitely differentiable, with $\hat{G}(\mathbf{k})$ also compactly supported inside a ball of radius 1 about the origin in Fourier space and with $G(\mathbf{s})$ decaying faster than any power $|\mathbf{s}|^{-p}$ as $|\mathbf{s}| \rightarrow \infty$. See for instance Appendix A in Eyink and Aluie (2009) for explicit examples. It can 
be shown that for any kernel $G(\mathbf{s})$ with the above properties, a coarse-grained function $\bar{f}_{\ell}(\mathbf{x})$ is infinitely differentiable 1

We can also define a complementary high-pass filter which retains only modes at scales $<\ell$ by

$$
\mathbf{a}_{\ell}^{\prime}(\mathbf{x})=\mathbf{a}(\mathbf{x})-\overline{\mathbf{a}}_{\ell}(\mathbf{x})
$$

In the rest of our paper, we shall take the liberty of dropping subscript $\ell$ whenever there is no risk of ambiguity.

It has been remarked by Sagaut and Germano (2005) and Garnier, Adams, and Sagaut (2009) that filtering a strong discontinuity in a field, such as an external shock from an explosion, introduces "parasitic" contributions which can overwhelm the turbulent fluctuations at small scales. Since our primary purpose in this work is a fundamental physical understanding rather than modeling of nonlinear scale interactions, and since such a strong shock would interact with the flow, we consider it only natural to include its contributions to sub-scales $<\ell$.

The filtering operation (6) is linear and commutes with space (and time) derivatives. We can apply it to the continuity and momentum equations (1)-(2) to describe dynamics of large-scale fields. However, as we mentioned above, there is no unique way to filter these equations. For example, we may define a large-scale momentum field either as $\bar{\rho}_{\ell} \overline{\mathbf{u}}_{\ell}$ or as $\overline{\rho \mathbf{u}} \ell$. Similarly, a large-scale kinetic energy may be defined as $\frac{1}{2} \bar{\rho}_{\ell}\left|\overline{\mathbf{u}}_{\ell}\right|^{2}$ or $\frac{1}{2}\left|\overline{\sqrt{\rho}}_{\ell}\right|^{2}$.

\section{Identifying the viscous range}

A key idea of this paper is that the scale-decomposition of momentum and kinetic energy should satisfy the inviscid criterion, i.e. it should guarantee that viscous contributions are negligible at large enough length-scales. This is necessary for the study of inertial range dynamics if such a scale-range exists in compressible turbulence.

\subsection{Scale decomposition}

Assume for simplicity that $\mu(\mathbf{x})=\mu$ is independent of spatial position $\mathbf{x}$ such as in the case of isothermal flows. We shall consider the more general case afterward. Coarse-graining eq. (2) and commuting the filter with space derivatives in the viscous diffusion terms yields

$$
\partial_{t} \overline{\rho u}_{i}+\partial_{j}\left(\overline{\rho u_{i} u_{j}}\right)=-\partial_{i} \bar{P}+\mu \partial_{j}\left\{\left(\partial_{j} \bar{u}_{i}+\partial_{i} \bar{u}_{j}\right)-\frac{2}{d} \partial_{k} \bar{u}_{k} \delta_{i j}\right\}+\overline{\rho F}_{i} .
$$

With such a decomposition, the functional form of viscous terms in 8 is similar to their counterpart in incompressible flows despite the additional contributions due to compressibility. If $u_{r m s}^{2}=$

\footnotetext{
${ }^{1}$ Under the very weak requirement that $\int_{\Omega} d \mathbf{x}|f(\mathbf{x})|<\infty$ over the domain $\Omega$ of the flow.
} 
$\int d \mathbf{x}|\mathbf{u}(\mathbf{x})|^{2}<\infty$, it can be shown rigorously that each of the viscous terms in eq. 8 is bounded by (const.) $\mu u_{r m s} / \ell^{2}(L / \ell)^{3 / 2}$ at every point $\mathbf{x}$. Therefore, the large-scale momentum, defined as $\bar{\rho}_{\ell}$, does not diffuse under the action of molecular viscosity when $\mu u_{r m s} / \ell^{2} \ll 1$. The type of proof used is standard in real analysis and for applications in turbulence theory, see Eyink (2007) and Aluie (2009). We detail the proof in A. Proposition 1. The idea behind it is simple and purely kinematic; a term $\mu \nabla^{2} \overline{\mathbf{u}}$ involves derivatives of a smooth filtered field. Therefore, such gradients cannot become arbitrarily large as $\mu \rightarrow 0$, even though unfiltered gradients, $\boldsymbol{\nabla u}$, may become unbounded. In fact, $\mu \nabla^{2} \overline{\mathbf{u}}$ may be expressed ${ }^{2}$ in terms of quantities at scale $\ell$, namely a big- $O$ bound $O\left(\mu \delta u(\ell) / \ell^{2}\right)$ which becomes negligible as $\mu \rightarrow 0$. Here, an increment is $\delta \mathbf{u}(\ell)=u(\mathbf{x}+\boldsymbol{\ell})-u(\mathbf{x})$.

The reader might question the value of a careful proof when one can arrive at the same conclusion by a simple dimensional argument. To illustrate the potential pitfalls of dimensional reasoning here, consider the quantity $\mu \overline{\nabla \mathbf{u}: \nabla \mathbf{u}_{\ell}}$. It may be argued that this should also vanish as $\mu \rightarrow 0$ for some fixed $\ell>0$. However, it is well known in turbulence literature that it does not (see for example Sreenivasan (1984, 1998); Pearson et al. (2004)). The problem lies in that $\mu \overline{\nabla \mathbf{u}: \boldsymbol{\nabla} \mathbf{u}_{\ell}}$ cannot be rewritten as derivatives of filtered fields. In other words, it cannot be expressed in terms of quantities at scale $\ell$, such as $O\left(\mu \delta u(\ell)^{2} / \ell^{2}\right)$, as one might innocently expect. When $\mu \rightarrow 0$ gradients can become unbounded and the term $\overline{\boldsymbol{\nabla u}: \boldsymbol{\nabla} \mathbf{u}_{\ell}}$ diverges. Phrased in the language of Fourier analysis, even though $\overline{\boldsymbol{\nabla u}: \boldsymbol{\nabla}} \mathbf{u}_{\ell}$ has small wavenumber modes $<K \sim \ell^{-1}$, it can be dominated by contributions from $\hat{\mathbf{u}}(\mathbf{q})$ with wavenumbers $|\mathbf{q}| \gg K$ due to the convolution $\boldsymbol{\nabla} \mathbf{u}: \boldsymbol{\nabla u}$ in Fourier space. Here,

$$
\widehat{\mathbf{u}}(\mathbf{q})=\int d \mathbf{x} \mathbf{u}(\mathbf{x}) e^{-i \mathbf{q} \cdot \mathbf{x}}
$$

is a Fourier transform. Put more explicitly, while the product $\widehat{\nabla \mathbf{u}}(\mathbf{q}): \widehat{\nabla \mathbf{u}}(\mathbf{k}-\mathbf{q}) e^{i \mathbf{q} \cdot \mathbf{x}} e^{i(\mathbf{k}-\mathbf{q}) \cdot \mathbf{x}}$ has a Fourier mode at wavenumber $|\mathbf{k}|<K$, it is proportional to $\sim|\mathbf{q}|^{2}$. This example has a direct bearing on our definition of large-scale momentum. If we were to define large-scale momentum as $\bar{\rho}_{\ell} \overline{\mathbf{u}}_{\ell}$ rather than $\overline{\rho \mathbf{u}}_{\ell}$ as we did above, a viscous term in the balance equation would have the form $\mu \bar{\rho}_{\ell} \overline{\rho^{-1} \nabla^{2} \mathbf{u}_{\ell}}$. Here, again, the filtering operation would not commute with the laplacian and, due to possibly dominant contributions from high wavenumber modes $\gg \ell^{-1}$, we would not be able to guarantee a prior $3^{3}$ that viscous terms are negligible at large $\ell$.

The arguments above and the proof in Proposition 1 presume that $\mu$ is a constant. In Proposition 3 we extend the result to the more general case of a spatially variable viscosity, $\mu(\mathbf{x})$, under an additional assumption that $\nabla \mu$ vanishes with $\mu \rightarrow 0$. In A.2, we provide a physical justification for this assumption based on Sutherland's law or a power law relation, $\mu(\mathbf{x}) \sim T^{\alpha}(\mathbf{x})$. A priori tests of compressible

\footnotetext{
${ }^{2}$ See remark following Proposition 1

${ }^{3}$ It is possible to prove that $\mu \bar{\rho}_{\ell} \bar{\rho}^{-1} \nabla^{2} \mathbf{u}_{\ell}$ vanishes with $\mu \rightarrow 0$ under very restrictive assumptions on smoothness of the density field. Put loosely, such assumptions would correspond to a density spectrum decaying faster than $k^{-3}$, which is physically unrealistic in highly compressible flows.
} 
turbulence simulations by Vreman, Geurts, and Kuerten (1995), Vreman (1995), and Martin, Piomelli, and Candler (2000) seem to suggest that, indeed, the additional non-linearity in viscous terms introduced by a spatially varying $\mu(\mathbf{x})$ is small.

The scale decompostion employed in the large-scale momentum balance (8) is equivalent to traditional Favre filtering (see for example Garnier et al. (2009)), where a Favre filtered function is weighted by the density:

$$
\widetilde{f}_{\ell}(\mathbf{x}) \equiv \frac{\overline{\rho f}_{\ell}(\mathbf{x})}{\bar{\rho}_{\ell}(\mathbf{x})} .
$$

The operator $(\widetilde{\cdot})$ is linear but does not commute with derivatives. The large-scale momentum balance (8) can be rewritten using definition (9) as

$$
\partial_{t} \bar{\rho} \widetilde{u}_{i}+\partial_{j}\left(\bar{\rho} \widetilde{u}_{i} \widetilde{u}_{j}\right)=-\partial_{j}\left(\bar{\rho} \widetilde{\tau}\left(u_{i}, u_{j}\right)\right)-\partial_{i} \bar{P}+\partial_{j} \bar{\sigma}_{i j}+\bar{\rho} \widetilde{F}_{i} .
$$

This is the same as the "bare" momentum equation (2) itself but with an additional contribution from turbulent stress,

$$
\bar{\rho} \widetilde{\tau}\left(u_{i}, u_{j}\right) \equiv \bar{\rho}\left(\widetilde{u_{i} u_{j}}-\widetilde{u}_{i} \widetilde{u}_{j}\right),
$$

which accounts for the effect of eliminated scales $<\ell$ and vanishes identically in the absence of fluctuations at those small scales. One can also obtain a continuity equation for large-scale density:

$$
\partial_{t} \bar{\rho}+\partial_{i}\left(\bar{\rho} \widetilde{u}_{i}\right)=0
$$

A main advantage of the filtering approach to analyzing turbulent flows is an ability to resolve the relevant physical processes both in scale and in space as is apparent from the balance eqs. (10), (12). They describe the evolution of large-scale momentum and large-scale density at every $\mathbf{x}$ in the flow and at variable resolution $\ell$. Using $\bar{\rho}_{\ell}$ and $\overline{\rho \mathbf{u}_{\ell}}$, and eqs. $(10),(12)$, it is also straightforward to derive a budget for kinetic energy density at scales $>\ell$, for arbitrary $\ell$. This yields

$$
\partial_{t} \bar{\rho}_{\ell} \frac{\left|\widetilde{\mathbf{u}}_{\ell}\right|^{2}}{2}+\nabla \cdot \mathbf{J}_{\ell}=-\Pi_{\ell}-\Lambda_{\ell}+\bar{P}_{\ell} \boldsymbol{\nabla} \cdot \overline{\mathbf{u}}_{\ell}-D_{\ell}+\epsilon_{\ell}^{i n j},
$$

where $\mathbf{J}_{\ell}(\mathbf{x})$ is space transport of large-scale kinetic energy, $\Pi_{\ell}(\mathbf{x})+\Lambda_{\ell}(\mathbf{x})$, which we examine closely in section 4 is usually called the subgrid scale (SGS) kinetic energy flux to scales $\left\langle\ell,-\bar{P}_{\ell} \boldsymbol{\nabla} \cdot \overline{\mathbf{u}}_{\ell}\right.$ is large-scale pressure dilatation, $D_{\ell}(\mathbf{x})$ is viscous dissipation acting on scales $>\ell$, and $\epsilon_{\ell}^{i n j}(\mathbf{x})$ is the energy 
injected due to external stirring. These terms are defined as

$$
\begin{aligned}
\Pi_{\ell}(\mathbf{x}) & =-\bar{\rho} \partial_{j} \widetilde{u}_{i} \widetilde{\tau}\left(u_{i}, u_{j}\right) \\
\Lambda_{\ell}(\mathbf{x}) & =\frac{1}{\bar{\rho}} \partial_{j} \bar{P} \bar{\tau}\left(\rho, u_{j}\right) \\
D_{\ell}(\mathbf{x}) & =\partial_{j} \widetilde{u}_{i}\left[2 \overline{\mu S_{i j}}-\frac{2}{d} \overline{\mu S_{k k}} \delta_{i j}\right] \\
J_{j}(\mathbf{x}) & =\bar{\rho} \frac{|\widetilde{\mathbf{u}}|^{2}}{2} \widetilde{u}_{j}+\bar{P} \bar{u}_{j}+\widetilde{u}_{i} \bar{\rho} \widetilde{\tau}\left(u_{i}, u_{j}\right)-\widetilde{u}_{i} \bar{\sigma}_{i j} \\
\epsilon_{\ell}^{i n j}(\mathbf{x}) & =\widetilde{u}_{i} \bar{\rho} \widetilde{F}_{i}
\end{aligned}
$$

where we employed in 15 the notation

$$
\bar{\tau}_{\ell}(f, g) \equiv \overline{(f g)_{\ell}}-\bar{f}_{\ell} \bar{g}_{\ell}
$$

for $2^{\text {nd }}$-order generalized central moments of any fields $f(\mathbf{x}), g(\mathbf{x})$ (see Germano (1992)).

Just as we have shown that viscous diffusion of large-scale momentum $\overline{\rho \mathbf{u}}=\bar{\rho} \widetilde{\mathbf{u}}$ is negligible, we can also rigorously prove under very weak conditions that viscous dissipation $D_{\ell}(\mathbf{x})$ of large-scale kinetic energy $\frac{1}{2} \bar{\rho}|\widetilde{\mathbf{u}}|^{2}$ vanishes at every point $\mathbf{x}$ when $\mu u_{r m s}^{2} / \ell^{2} \ll 1$. The rigorous proofs for both cases of a constant and spatially varying $\mu$ are given in Propositions 2 and 4 of $\mathrm{A}$, respectively.

\subsection{Favre filtering and the inviscid criterion}

In one of his original articles Favre (1969), Favre motivated the usage of density-weighted averaging by the fact that average mass of a fluid in a volume $V$ advected by the large-scale velocity $\langle\rho \mathbf{u}\rangle /\langle\rho\rangle$ is conserved We shall now briefly repeat Favre's argument. The change of average mass in a volume $V$ advected with some large-scale velocity $\mathbf{u}^{*}$ is

$$
\int_{V} d^{3} \mathbf{x} \partial_{t}\langle\rho\rangle+\nabla \cdot\left(\langle\rho\rangle \mathbf{u}^{*}\right)=\int_{V} d^{3} \mathbf{x} \nabla \cdot\left(\langle\rho\rangle \mathbf{u}^{*}-\langle\rho \mathbf{u}\rangle\right),
$$

where now $\langle\ldots\rangle$ denotes ensemble averaging. The equality follows from using the ensemble averaged continuity eq. (1). Favre averaged velocity $\widetilde{\mathbf{u}}$ is defined as the choice of $\mathbf{u}^{*}$ which makes average flux of mass due to fluctuations (or turbulence) vanish, $\langle\rho\rangle \widetilde{\mathbf{u}}-\langle\rho \mathbf{u}\rangle:=0$. The same argument carries over to spatially filtered dynamics, where now $\widetilde{\mathbf{u}}_{\ell}$ is defined as the choice of large-scale velocity which does not lead to subgrid terms in the filtered continuity eq. 12.

${ }^{4}$ There were other factors in Favre's choice of "Méthode $\langle\mathrm{B}\rangle$," as he termed it, including a simpler form of the resultant equations, and applicability to experimental measurements (e.g. see Gatski and Bonnet (2009) for an overview). 
There are two comments we would like to make concerning Favre's argument. First, the special property large-scale velocity $\widetilde{\mathbf{u}}$ enjoys, i.e. suppressing turbulent diffusion of mass in eq. (12), does not logically imply by itself that a density-weighted decomposition is a necessary choice in the mass balance 12. It is certainly not unphysical for a turbulent flow to diffuse mass and, in this respect, the choice of a large-scale velocity field would depend on the particular purpose of an investigation. Second, a criterion requiring that turbulent mass diffusion be zero has no logical implication on the scale decomposition of momentum and kinetic energy. Unlike in eq. 12, the Favre decomposition results in turbulent diffusion and dissipation of large-scale momentum and kinetic energy as seen from eqs. 10,, 13 .

Yet, density-weighted filtering is used extensively in LES of compressible turbulence due to its modeling appeal. One of the perceived advantages is the absence of subgrid terms to be modeled in the coarse-grained continuity eq. (12). Another reason is that Favre filtered equations $[10,,(13)$ are structurally similar to their classically filtered counterparts in incompressible flows, which allows for carrying over models from the incompressible LES literature. Furthermore, none of the subgrid scale terms is a function of pressure which practitioners try to avoid modeling. When using the ideal gas law, $P=$ (const.) $\rho T$, as the equation of state, there is also an added advantage that filtered pressure, $\bar{P}$, can be expressed as a function of resolved quantities, $\bar{\rho}$ and $\widetilde{T}$, without additional subgrid terms.

What we have shown above is that Favre decomposition of momentum and kinetic energy satisfies the inviscid criterion. It guarantees that viscous contributions are negligible at large enough lengthscales. Such a decomposition of momentum and kinetic energy is borne out of a physical requirement, irrespective of practical modeling considerations. We remark, however, that it may not be the unique decomposition satisfying the inviscid criterion. In other words, we did not prove that it is necessary. We only showed that the Favre decomposition is sufficient to satisfy the inviscid criterion.

As we mentioned in the introduction, while our equations $\sqrt{10}, \sqrt{12}, \sqrt{13})$ coincide to a considerable extent with those that are employed in LES of compressible turbulence, their use here and in the ensuing papers will be for rather different purposes. Whereas the primary goal in LES is to model the subgrid terms, the aim here is to develop a physical understanding of these terms and estimate their contributions at different scales, including limits of small $\ell \ll L$, through exact mathematical analysis.

\section{Inertial dynamics}

Now that we have isolated viscous effects to the smallest scales $\ell_{\mu}$, where $\ell_{\mu}$ is defined as the scale at which viscous effects become significant in kinetic energy balance 13 , , we can study the dynamics at scales $\ell \gg \ell_{\mu}$. 


\subsection{Deformation work}

The first term in kinetic energy SGS flux, $\Pi_{\ell}$ in eq. 13 , is the contribution from deformation work done by large-scale strain $\partial_{j} \widetilde{u}_{i}$ against the subgrid stress $\bar{\rho} \widetilde{\tau}\left(u_{i}, u_{j}\right.$ ) (see for example Tennekes and Lumley (1972)). This is similar to its incompressible counterpart except that the strain is not traceless here. It acts as a sink in the large-scale kinetic energy budget (13) and as source in the complementary smallscale kinetic budget (51), and represents that part of the kinetic energy transferred from scales larger than $\ell$ to smaller scales at point $\mathbf{x}$ in the flow.

Furthermore, $\Pi_{\ell}(\mathbf{x})$ is Galilean invariant due to the subtracted large-scale terms in definition (11) of the turbulent stress. Other definitions of the SGS flux are possible such as $\widetilde{u}_{i} \partial_{j}\left(\bar{\rho} \widetilde{\tau}\left(u_{i}, u_{j}\right)\right)$ which differs from our definition (14) by a total gradient $\partial_{j}\left(\bar{\rho} \widetilde{u}_{i} \widetilde{\tau}\left(u_{i}, u_{j}\right)\right)$. However, this definition is not pointwise Galilean invariant, so the amount of "energy cascade" at any point $\mathbf{x}$ in the fluid according to such a definition would depend on the observer's velocity. Kraichnan Kraichnan (1964), Speziale Speziale (1985), and Germano Germano (1992) all emphasized the importance of Galilean invariance. More recently, Eyink and Aluie Eyink and Aluie (2009); Aluie and Eyink (2009, 2010) showed that Galilean invariance was necessary for scale-locality of the cascade. There are non-Galilean-invariant terms in our budget (13) but, as is physically natural, they are all associated with space transport $\mathbf{J}$ of kinetic energy.

Another physical requirement on the flux $\Pi_{\ell}(\mathbf{x})$ is that it should vanish in the absence of fluctuations at scales smaller than $\ell$ (or a moderate fraction thereof); for example, when $\ell$ is equal to $2 \pi / K_{\max }$, where $K_{\max }$ is the maximum wavenumber in a numerical simulation Aluie and Kurien (2011). This is satisfied by our definition of $\Pi_{\ell}(\mathbf{x})$ identically at every point $\mathbf{x}$ in the flow. Other definitions of an energy flux are possible, such as the "unsubtracted flux" of an incompressible flow critiqued in Eyink and Aluie (2009); Aluie and Eyink (2009),

$$
\Pi_{\ell}^{u n s}(\mathbf{x}) \equiv \bar{u}_{i} \overline{\mathcal{N L}}_{i}, \quad \mathcal{N} \mathcal{L}_{i}=\partial_{j}\left(\rho u_{i} u_{j}\right)
$$

which is often employed in literature that considers the sharp-spectral filter. Here, $\mathcal{N} \mathcal{L}_{i}$ denotes the nonlinearity in the momentum equation.

Using such a filter, the "unsubtracted flux" across wavenumber $K$ is computed as

$$
\Pi_{K}^{\text {uns }}(\mathbf{x})=\sum_{|\mathbf{p}|<K} \hat{u}_{i}(\mathbf{p}) e^{i \mathbf{p} \cdot \mathbf{x}} \sum_{|\mathbf{k}|<K} \widehat{\mathcal{N L}}_{i}(\mathbf{k}) e^{i \mathbf{k} \cdot \mathbf{x}} .
$$

Taking $K=K_{\max }$, we have $\Pi_{K_{\max }}^{u n s}(\mathbf{x})=u_{i} \partial_{j}\left(\rho u_{i} u_{j}\right)$ which is in general nonzero. It is only after averaging over all space (and in the absence of flow beyond the domain boundary) that one gets

$$
\left\langle\Pi_{K_{\text {max }}}^{u n s}\right\rangle=\left\langle\partial_{j}\left(\rho \frac{|\mathbf{u}|^{2}}{2} u_{j}\right)\right\rangle=\sum_{|\mathbf{k}|<K_{\text {max }}} \hat{u}_{i}^{*}(\mathbf{k}) \widehat{\mathcal{N L}}_{i}(\mathbf{k})=0 .
$$


Similar considerations apply for compressible flows, where an unsubtracted flux may be defined as

$$
\Pi_{\ell}^{u n s}(\mathbf{x}) \equiv \widetilde{u}_{i} \overline{\mathcal{N}}_{i}-\frac{1}{2}|\widetilde{\mathbf{u}}|^{2} \overline{\mathcal{N}}, \quad \mathcal{N} \mathcal{L}_{i}=\partial_{j}\left(\rho u_{i} u_{j}\right), \quad \mathcal{N}=\partial_{j}\left(\rho u_{j}\right)
$$

with $\mathcal{N} \mathcal{L}_{i}$ and $\mathcal{N}$ denoting the nonlinearities in momentum and density equations, respectively. This flux does not vanish in general when $\ell=K_{m a x}^{-1}$, except after space-averaging.

\subsection{Baropycnal work}

The other part of kinetic energy flux, $\Lambda_{\ell}$ in eq. (13), is intrinsically due to compressibility effects and vanishes in the absence of density variations. It represents work done by a large-scale pressure-gradient forc $\sum^{5} \bar{\rho}^{-1} \nabla \bar{P}$ against subscale mass flux ${ }^{6} \bar{\tau}(\rho, \mathbf{u})$. We shall refer to $\Lambda_{\ell}(\mathbf{x})$ as baropycnal work due to its inherent dependence on pressure and density variations. It is not entirely due to baroclinic effects for it can be non-zero even when small-scale density variations and $\nabla \bar{P}_{\ell}$ are aligned as shown in Figure 1 . Similar to $\Pi_{\ell}$, it also acts as a sink in the large-scale kinetic energy budget (13) and as a source in the small-scale budget (51), is pointwise Galilean invariant, and vanishes identically at every $\mathbf{x}$ in the absence of fluctuations at scales $<\ell$. Baropycnal work is known to play a major role in turbulent combustion (see for example Starner and Bilger (1980); Libby and Bray (1981)). It has also been recently observed in Livescu and Ristorcelli (2007); Livescu et al. (2009) to play a major role in turbulence production in buoyancy-driven flows with significant density differences. In such flows, termed "variable-density flows" in Livescu and Ristorcelli (2007), there are two or more incompressible miscible fluid species, such as water and brine, which have significantly different densities.

The physical mechanism behind this part of the SGS flux, illustrated in Figure 1, is simple. In a frame co-moving with a ball of radius $\ell$ in the flow, a pressure-gradient force $\bar{\rho}^{-1} \nabla \bar{P}_{\ell}$ at scales $>\ell$ acts on the ball within which the fluid has non-uniform density (density variations at scales $<\ell$ ). Per unit time, if $\rho_{2}>\rho_{1}$ in Figure 1 and $\left|\mathbf{u}_{1}\right|=\left|\mathbf{u}_{2}\right|=u$, then parcel 2 gains $\bar{\rho}^{-1}\left|\nabla \bar{P}_{\ell}\right| \rho_{2} u$ in kinetic energy from the large-scales and parcel 1 loses $\bar{\rho}^{-1}\left|\nabla \bar{P}_{\ell}\right| \rho_{1} u$ to the large-scales. On aggregate, scales $<\ell$ in this ball would gain kinetic energy from the large-scales at rate, $\bar{\rho}^{-1}\left|\nabla \bar{P}_{\ell}\right|\left(\rho_{2}-\rho_{1}\right) u$. This effect would vanish in the absence of density variations. Note that if $\left|\mathbf{u}_{1}\right|$ does not equal $\left|\mathbf{u}_{2}\right|$, then the whole ball will have an average large-scale velocity $\overline{\mathbf{u}}_{\ell}=\mathbf{u}_{1}+\mathbf{u}_{2}$ which does not play a role in such a process of inter-scale energy transfer - hence the relevance of the premise of a co-moving frame.

Similar to deformation work, $\Pi_{\ell}(\mathbf{x})$, baropycnal work, $\Lambda_{\ell}(\mathbf{x})$, is not sign-definite. We expect that at points $\mathbf{x}$ in the flow where large-scale pressure gradient $\nabla \bar{P}_{\ell}(\mathbf{x})$ opposes the density gradient due to small-scale fluctuations $\left(\rho_{2}>\rho_{1}\right.$ in Figure 1), then a motion similar to that of a Rayleigh-Taylor instability would ensue such that velocities $\mathbf{u}_{1}$ and $\mathbf{u}_{2}$ will be as illustrated in Figure 1. We, therefore,

\footnotetext{
${ }^{5}$ The term "pressure-gradient force" is used in the meteorology literature. It is not a force but an acceleration.

${ }^{6}$ Here, "flux" denotes a flux in space, not in scale.
} 


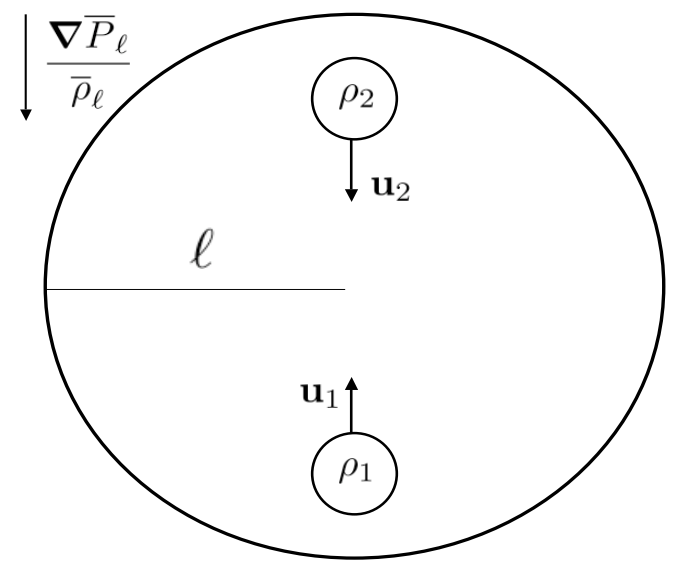

Figure 1: Heuristic explanation of the physics behind baropycnal work, $\Lambda_{\ell}$. In a ball of radius $\ell$, there are two small-scale fluid parcels of equal size with densities $\rho_{1}$ and $\rho_{2}$, and antiparallel velocities $\mathbf{u}_{1}$ and $\mathbf{u}_{2}$, respectively. In a frame co-moving with the ball, we must have $\left|\mathbf{u}_{1}\right|=\left|\mathbf{u}_{2}\right|$.

expect that at such points in the flow $\Lambda_{\ell}(\mathbf{x})$ will be positive and transfer energy to small-scales. On the other hand, in regions such as shocks where large-scale pressure gradient is in the same direction as the density gradient due to small-scale fluctuations $\left(\rho_{2}<\rho_{1}\right.$ in Figure 1), we expect that $\Lambda_{\ell}(\mathbf{x})$ will be negative and transfer energy to large-scales. Whether, on average, the subscale mass flux $\bar{\tau}(\rho, \mathbf{u})$ would correlate positively or negatively with large-scale acceleration field $\bar{\rho}^{-1} \nabla \bar{P}$ can be determined empirically.

Despite the recognition of $\Lambda_{\ell}$ 's importance in turbulent combustion and variable-density flows, to the best of our knowledge, this term has never been studied as a function of scale $\ell$. The presence of baropycnal work as a cascade mechanism to transfer kinetic energy between scales has not been appreciated in the literature. Possibly due to Favre's original formulation Favre (1969) (see also a review by Lele Lele $(1994)$ ), this term has often been lumped with $\bar{P}_{\ell} \boldsymbol{\nabla} \cdot \overline{\mathbf{u}}_{\ell}$ in the form $\bar{P}_{\ell} \boldsymbol{\nabla} \cdot \widetilde{\mathbf{u}}_{\ell}$ (plus an additional space-transport term) and treated as a large-scale pressure dilatation which does not require modeling. An exception is the work by Huang et al. Huang et al. (1995) who, from a modeling standpoint, espoused separating the two terms $\left(\Lambda_{\ell}\right.$ and $\left.\bar{P}_{\ell} \boldsymbol{\nabla} \cdot \overline{\mathbf{u}}_{\ell}\right)$ as we have done in eq. 13) on the belief that a density-weighted decomposition should only be applied to the convective terms. In contrast to Huang et al. (1995), our reason for keeping baropycnal work separate from pressure dilatation is due to a fundamental distinction between the two. Notice that both deformation work, $\Pi_{\ell}$, and baropycnal work, $\Lambda_{\ell}$, involve large-scale fields acting against small-scale fluctuations. This makes them capable of transferring energy across scales. On the other hand, large-scale pressure dilatation, $\bar{P}_{\ell} \boldsymbol{\nabla} \cdot \overline{\mathbf{u}}_{\ell}$, involves only large-scales and cannot transfer energy directly across scales. 


\subsection{Pressure dilatation}

Large-scale pressure dilatation $-\bar{P} \nabla \cdot \overline{\mathbf{u}}$ in eq. 13 represents conversion of large-scale kinetic energy to internal energy through compression. In the incompressible limit, this vanishes at every point $\mathbf{x}$. Unlike $\Pi_{\ell}$ and $\Lambda_{\ell}$, pressure dilatation only contains scales $>\ell$ (at least for filter kernels $\hat{G}(\mathbf{k})$ compact in Fourier space). Therefore, it is not involved in the transfer of energy across scale $\ell$ and it does not vanish in the absence of subscale fluctuations. We recently analyzed this term using data from highresolution numerical simulations Aluie et al. (2012) and concluded that pressure dilatation acts primarily at large-scales, on average.

\section{$5 \quad$ Kinetic energy injection}

Similar to localizing viscous dissipation to the smallest scales in a flow, localizing kinetic energy injection to the largest scales is just as important to enable the study of an intermediate scale-range over which inertial processes dominate.

When density is constant, $\rho=\rho_{0}$, both kinetic energy, $\rho_{0}|\mathbf{u}|^{2} / 2$, and its injection, $\epsilon^{i n j}=\rho_{0} \mathbf{u} \cdot \mathbf{F}$, are quadratic quantities. This allows kinetic energy injection to be easily localized to the largest scales by restricting $\mathbf{F}$ to small Fourier wavenumbers as is commonly done. To recap why that this, we will denote a field in a $d$-dimensional periodic domain $\mathbb{T}^{d}=[0,2 \pi)^{d}$, coarse-grained with the sharp-spectral filter to retain only Fourier modes $|\mathbf{k}|<K$, by

$$
\mathbf{a}^{<K}(\mathbf{x}) \equiv \sum_{|\mathbf{k}| \leq K} \hat{\mathbf{a}}(\mathbf{k}) e^{i \mathbf{k} \cdot \mathbf{x}}
$$

This is similar to $\overline{\mathbf{a}}_{\ell}(\mathbf{x})$ with $\ell$ being of the same order as $K^{-1}$. Now consider an imposed acceleration $\mathbf{F}^{<K_{0}}$ with modes restricted to small wavenumbers $\leq K_{0}$. In an incompressible flow, mean "large-scale" kinetic energy at modes $\leq K$ is $\rho_{0}\left|\mathbf{u}^{<K}\right|^{2} / 2$, and the mean injection into those modes reduces to

$$
\rho_{0}\left\langle\mathbf{u}^{<K} \cdot \mathbf{F}^{<K_{0}}\right\rangle=\rho_{0}\left\langle\mathbf{u}^{<K_{0}} \cdot \mathbf{F}^{<K_{0}}\right\rangle
$$

due to orthogonality of Fourier modes. Relation (22) shows that mean injection into modes $<K$ becomes independent of $K$ for $K \geq K_{0}$, implying that there is no net energy deposited by the forcing at modes $\geq K_{0}$. In other words, relation (22) shows kinetic energy is injected at wavenumbers $\leq K_{0}$ when $\mathbf{F}$ is restricted to modes $\leq K_{0}$. In the more general case of variable density flows, relation 22] no longer holds and localizing the injection, now a cubic quantity, is not as obvious.

While most numerical simulations of forced compressible turbulence stir the flow with a large-scale acceleration of the form $\mathbf{F}^{<K_{0}}$ (e.g. Kida and Orszag (1990); Schmidt et al. (2009); Petersen and Livescu (2010); Federrath et al. (2010); Kritsuk et al. (2007)), to the best of our knowledge, the first study to 
pose the issue of whether such stirring restricts kinetic energy injection to the largest scales was by Wagner et al. Wagner et al. (tion). However, the authors of Wagner et al. (tion) did not investigate injection of kinetic energy but that of $\left\langle|\rho \mathbf{u}|^{2}\right\rangle / 2$.

We shall now show analytically that stirring with an acceleration field of the form $\mathbf{F}^{<K_{0}}(\mathbf{x})$ restricts kinetic energy injection to the largest scales (within the Favre scale-decomposition framework utilized in budget 13 , where "large-scale" for kinetic energy is $\bar{\rho}|\widetilde{\mathbf{u}}|^{2} / 2$ ). We defer the rigorous proof to $\mathrm{B}$, but the logic behind it is simple and goes as follows.

$$
\begin{aligned}
\left\langle\epsilon_{\ell}^{i n j}\right\rangle & =\left\langle\frac{\bar{\rho} \overline{\mathbf{u}}_{\ell}}{\bar{\rho}_{\ell}} \cdot \overline{\rho \mathbf{F}}_{\ell}\right\rangle \\
& =\left\langle\frac{\bar{\rho} \overline{\mathbf{u}}_{\ell}}{\bar{\rho}_{\ell}} \cdot \bar{\rho}_{\ell} \overline{\mathbf{F}}_{\ell}\right\rangle+\left\langle\frac{\overline{\rho \mathbf{u}}_{\ell}}{\bar{\rho}_{\ell}} \cdot \bar{\tau}_{\ell}(\rho, \mathbf{F})\right\rangle,
\end{aligned}
$$

which is exact. When the forcing $\mathbf{F}(\mathbf{x})=\mathbf{F}^{<K_{0}}(\mathbf{x})$ varies at very large scales $L \sim K_{0}^{-1} \gg \ell$, we have $\overline{\mathbf{F}}_{\ell}(\mathbf{x}) \approx \overline{\mathbf{F}}_{L}(\mathbf{x})$. Moreover, $\bar{\tau}_{\ell}(\rho, \mathbf{F})$, representing sub-scale fluctuations in $\rho$ and $\mathbf{F}$ at scales smaller than $\ell \ll L$, is negligible primarily because $\mathbf{F}(\mathbf{x})$ has no fluctuations at small scales. It follows that

$$
\left\langle\epsilon_{\ell}^{i n j}\right\rangle \approx\left\langle\bar{\rho} \mathbf{u}_{\ell} \cdot \overline{\mathbf{F}}_{L}\right\rangle \approx\left\langle(\rho \mathbf{u})^{<K_{0}} \cdot \mathbf{F}^{<K_{0}}\right\rangle=\left\langle\epsilon_{K_{0}}^{i n j}\right\rangle,
$$

where the third expression follows from the orthogonality of Fourier modes. In B we present a rigorous proof of statement (24) under precise (and weak) conditions. Relation (24) shows that mean injection into scales $>\ell$ becomes independent of $\ell$ for $\ell \ll K_{0}^{-1}$. Result 24 also implies that injection at scales smaller than $\ell,\left\langle\epsilon_{\ell}^{\text {small }}\right\rangle$ in eq. (54), vanishes as $\ell K_{0} \rightarrow 0$. This proves the non-trivial possibility to make injection localized to the largest scales in variable density flows by employing an external acceleration field limited to wavenumbers $\leq K_{0}$. Finally, we note that the acceleration field $\mathbf{F}^{<K_{0}}$ in momentum eq. (2) is of a general form and can have both solenoidal and irrotational components.

In arriving at eq. (24), we had to stir with an external acceleration field such that the force in (2) is weighted by instantaneous density $\rho(\mathbf{x}, t)$. Had we stirred the momentum equation with an external force $\mathcal{F}^{<K_{0}}$ instead of $\rho \mathbf{F}^{<K_{0}}$, the injection would have had the form $\left\langle\epsilon_{\ell}^{i n j}\right\rangle=\left\langle\widetilde{\mathbf{u}}_{\ell} \cdot \mathcal{F}^{<K_{0}}\right\rangle$ In B Proposition 6 . we prove rigorously that it converges to $\left\langle\mathbf{u}^{<K_{0}} \cdot \mathcal{F}^{<K_{0}}\right\rangle$, for $\ell K_{0} \rightarrow 0$. However, the rate of convergence according to our bound (47) is slower than that in 40, obtained by stirring with an acceleration field. This suggests that stirring with an acceleration field would yield a longer intertial range in numerical simulations with significant density fluctuations, compared to stirring with a force.

\subsection{The inertial range}

Following our result (24) and assuming statistically steady-state conditions, the spatially averaged largescale kinetic energy budget 13 at scales $K_{0}^{-1} \gg \ell \gg \ell_{\mu}$ becomes

$$
\left\langle\Pi_{\ell}\right\rangle+\left\langle\Lambda_{\ell}\right\rangle-\left\langle\bar{P}_{\ell} \boldsymbol{\nabla} \cdot \overline{\mathbf{u}}_{\ell}\right\rangle=\left\langle\epsilon_{K_{0}}^{i n j}\right\rangle=\text { (const.), }
$$


such that the sum of kinetic energy flux and pressure dilatation on the left hand side is constant, independent of scale $\ell=K^{-1}$. In relation 25$]$, we assumed that none of the kinetic energy is transported beyond the domain boundary. We also dropped viscous dissipation, $\left\langle D_{\ell}\right\rangle$, which we have proved to be negligible.

Relation 25 in itself is not an analogue to Kolmogorov's 4/5-th law since it contains the pressure dilatation term which does not involve energy transfer across scales. However, we have recently presented a sufficient condition along with a physical argument in Aluie (2011) which imply that $\left\langle\bar{P}_{\ell} \boldsymbol{\nabla} \cdot \overline{\mathbf{u}}_{\ell}\right\rangle$ acts primarily on the largest scales, similar to $\left\langle\epsilon_{K_{0}}^{i n j}\right\rangle$. We have also shown evidence from numerical simulations in Aluie et al. (2012) in support of this, i.e.

$$
\left\langle\bar{P}_{\ell} \boldsymbol{\nabla} \cdot \overline{\mathbf{u}}_{\ell}\right\rangle \approx\left\langle\bar{P}_{L_{c}} \boldsymbol{\nabla} \cdot \overline{\mathbf{u}}_{L_{c}}\right\rangle
$$

where $L_{c}$ is a large "conversion" length-scale similar to an integral scale. It follows that most of the net conversion between kinetic and internal energy takes place at scales larger than $L_{c}$ and equation 25. becomes

$$
\left\langle\Pi_{\ell}\right\rangle+\left\langle\Lambda_{\ell}\right\rangle=\left\langle\bar{P}_{L_{c}} \boldsymbol{\nabla} \cdot \overline{\mathbf{u}}_{L_{c}}\right\rangle+\left\langle\epsilon_{K_{0}}^{i n j}\right\rangle=\text { (const.) }
$$

over the scale-range $\ell_{\mu} \ll \ell \ll L_{c}$. A scale-independent kinetic energy flux in eq. (27) implies that kinetic energy cascades conservatively in a manner similar to energy cascade in incompressible turbulence. We presented evidence of such a cascade in Aluie et al. (2012). Relation 27) can be regarded as an analogue to Kolmogorov's 4/5-th law for compressible turbulence.

\subsection{Special case: Rayleigh-Taylor flow}

We shall apply our result on localized injection to the case of a Rayleigh-Taylor flow driven by gravitational forces. We ask the following question: At what scales does mean conversion of gravitational potential energy to kinetic energy take place?

We shall show that, in the presence of a spatially uniform gravitational field $\mathbf{g}(\mathbf{x})=\mathbf{g}$, such conversion into kinetic energy only takes place at the largest scale - that of the domain size. Consider the largescale kinetic energy equation 13 where $\mathbf{F}(\mathbf{x})$ is replaced with $\mathbf{g}$ in $\epsilon_{\ell}^{i n j}$. We have from 18 that net input of kinetic energy due to gravity is

$$
\left\langle\epsilon_{\ell}^{i n j}\right\rangle=\left\langle\widetilde{\mathbf{u}}_{\ell} \cdot \bar{\rho}_{\ell} \widetilde{\mathbf{g}}_{\ell}\right\rangle=\left\langle\bar{\rho}_{\ell} \cdot \mathbf{g}\right\rangle=\mathbf{g} \cdot\langle\rho \mathbf{u}\rangle
$$

where the last equality follows from $\int d \mathbf{x} \bar{f}_{\ell}(\mathbf{x})=\int d \mathbf{r} G_{\ell}(\mathbf{r}) \int d \mathbf{x} f(\mathbf{x}+\mathbf{r})=\langle f\rangle$. Result 28) shows that mean injection is independent of scale $\ell$ and only takes place at the scale of the domain, $L_{\mathrm{dom}}$.

Put in more detail, the mean kinetic energy $\left\langle\bar{\rho}_{\ell}\left|\widetilde{\mathbf{u}}_{\ell}\right|^{2}\right\rangle / 2$ at scales $>\ell$ increases at a rate $\left\langle\widetilde{\mathbf{u}}_{\ell} \cdot \bar{\rho}_{\ell} \widetilde{\mathbf{g}}_{\ell}\right\rangle$ due to gravitational forces. Consider a sequence of scales $\ell_{1}>\ell_{2}>\cdots>\ell_{n}>\ldots$ and the average 
rate of potential energy being converted into mean kinetic energy, $\left\langle\epsilon_{\ell_{n}}^{i n j}\right\rangle \equiv\left\langle\widetilde{\mathbf{u}}_{\ell_{n}} \cdot \bar{\rho}_{\ell_{n}} \widetilde{\mathbf{g}}_{\ell_{n}}\right\rangle$, at successively larger sets of scales $\left[\ell_{n}, L_{\mathrm{dom}}\right)$. In general, as $n \rightarrow \infty,\left\langle\epsilon_{\ell_{n}}^{i n j}\right\rangle$ approaches the total rate of potential energy converted into kinetic form. The fact that $\left\langle\epsilon_{\ell_{n}}^{i n j}\right\rangle$ is independent of $\ell_{n}$ over the entire scale-range [0, $\left.L_{\mathrm{dom}}\right)$ implies that all conversion takes place at the domain scale.

Our argument demonstrates the power of the filtering approach ${ }^{7}$ in analyzing non-linear scale interactions. We were able to arrive at our answer precisely because the filtering technique allows for probing a continuous range of scales, in contrast to the traditional averaging approach.

The conclusion is probably non-intuitive at face value because, in a Rayleigh-Taylor flow, "fingers" of heavy fluid at any scale penetrate the lighter fluid as they descend, converting potential to kinetic energy. It seems to contradict our result that conversion only takes place at the domain scale. Key to understanding such an ostensible paradox is the definition of kinetic energy based on a Favre scaledecomposition. Mean kinetic energy at scale $\ell$ may be rewritten as $\left\langle\left|\bar{\rho}_{\ell}\right|^{2} / \bar{\rho}_{\ell}\right\rangle / 2$, which emphasizes the central role of momentum in defining scale. Indeed, result (28) demonstrates that it is only the $k=0$ mode of momentum which participates in converting potential energy into kinetic energy at scale with mode $k=0,\langle\rho \mathbf{u}\rangle^{2} /\langle\rho\rangle / 2$. While momentum at scale $\ell$ can have contributions from density and velocity at all scales - for example, mean momentum $\langle\rho \mathbf{u}\rangle=\sum_{\mathbf{k}} \hat{\rho}(\mathbf{k}) \hat{\mathbf{u}}(-\mathbf{k})$ - the scale $\ell$ of kinetic energy depends on that of momentum $\overline{\rho \mathbf{u}}_{\ell}$ and not on, for instance, $\bar{\rho}_{\ell} \overline{\mathbf{u}}_{\ell}$.

\section{Compressibility effects in the SGS flux}

Our scale-decomposition allowed us to identify two SGS sinks for the large-scale kinetic energy budget, namely deformation work, $\Pi_{\ell}$, and baropycnal work, $\Lambda_{\ell}$.

While $\Pi_{\ell}$ also represents a cascade mechanism in incompressible turbulence, $\Lambda_{\ell}$ emerges from density fluctuations and thus is intrinsic to flows with variable density. However, even deformation work has contributions from compressibility effects. We have shown in Aluie $(2011 ?)$ that $\Pi_{\ell}=\bar{\rho} \partial_{j} \bar{u}_{i} \bar{\tau}\left(u_{i}, u_{j}\right)+$ $\ldots 8$ terms $\ldots$, using exact identities. If we consider that part of $\Pi_{\ell}$ equal to $\bar{\rho} \partial_{j} \bar{u}_{i} \bar{\tau}\left(u_{i}, u_{j}\right)$, we see that compressive modes with $\mathbf{k} \cdot \hat{\mathbf{u}}(\mathbf{k}) \neq 0$ can play an important role in deformation work. This is because the large-scale strain $\boldsymbol{\nabla} \overline{\mathbf{u}}$ is not traceless. The effect of compressive modes is best illustrated by the one-dimensional Burger's flow,

$$
\partial_{t} u+\partial_{x}\left(\frac{u^{2}}{2}\right)=\nu \partial_{x x} u
$$

A large-scale kinetic energy budget analogous to 13 can be derived (see for example Eyink (2007)):

$$
\partial_{t} \frac{|\bar{u}|^{2}}{2}+\partial_{x}\left\{\frac{\bar{u}^{3}}{3}+\bar{u} \frac{\bar{\tau}(u, u)}{2}-\nu \partial_{x} \frac{|\bar{u}|^{2}}{2}\right\}=-\Pi^{\text {burg }}-\nu\left|\partial_{x} \bar{u}\right|^{2},
$$

\footnotetext{
${ }^{7}$ In this particular case, with a constant $\mathbf{g}$ containing only a $k=0$ mode, the same conclusion would have also been possible by examining the injection, $\left\langle\epsilon_{\ell}^{\text {small }}\right\rangle$ in eq. $\sqrt[54]{ }$, into mean subscale kinetic energy, $\left\langle\overline{\rho|\mathbf{u}|^{2}}{ }_{\ell}-\bar{\rho}_{\ell}\left|\widetilde{\mathbf{u}}_{\ell}\right|^{2}\right\rangle / 2$, which may be easily determined to equal zero at any $\ell$. See C
} 
in which viscous dissipation on the right-hand-side is negligible and

$$
\Pi^{\text {burg }} \equiv-\partial_{x} \bar{u} \frac{\bar{\tau}(u, u)}{2}
$$

is the only sink. There are no shearing motions in flow 290 . The only way energy cascades to small scales is through $\Pi^{\text {burg }}$ which is solely due to compressive modes. As is well-known, this cascade is manifested in the formation of shocks.

In general, a simple measure that quantifies kinematic role of compressibility on deformation work (14) is

$$
\Pi_{\ell}^{c o m p} \equiv \Pi_{\ell}+\bar{\rho} \partial_{j} \bar{u}_{i}^{s} \bar{\tau}\left(u_{i}^{s}, u_{j}^{s}\right)
$$

where the velocity $\mathbf{u}=\mathbf{u}^{s}+\mathbf{u}^{c}$ is decomposed into solenoidal and irrotational components, $\mathbf{u}^{s}$ and $\mathbf{u}^{c}$, respectively.

\section{Summary}

In this paper we have shown that viscous diffusion and dissipation can be isolated to the smallest scales in a compressible flow by using a proper scale-decomposition. Guided by this physical requirement, which we call the "inviscid criterion", we found that a Favre decomposition of the momentum and kinetic energy into large-scale and small-scale components is sufficient to guarantee a negligible role of molecular viscosity in the large-scale dynamics of high Reynolds number flows.

We were also able to establish through an exact analysis that mean kinetic energy injection can be made localized to the largest scales in a flow by proper stirring. Moreover, our analysis suggested that stirring with an external acceleration field is more adequate to realizing a longer inertial range in a numerical simulation compared to stirring with an external force. We discussed the special case of buoyancy-driven flows in which stirring is due to a spatially-uniform gravitational field, and showed that mean injection of kinetic energy occurs only at the very largest scale in the system.

Localizing viscous dissipation to the smallest scales and energy injection to the largest scales is necessary to allow for studying inertial dynamics at intermediate scales. Under steady-state conditions, satisfying these two ingredients implies that the sum of mean SGS kinetic energy flux and pressure dilatation, $\left\langle\Pi_{\ell}+\Lambda_{\ell}\right\rangle-\left\langle\bar{P}_{\ell} \nabla \cdot \overline{\mathbf{u}}_{\ell}\right\rangle$, is constant, independent of scale $\ell$ over the intermediate range $L \gg$ $\ell \gg \ell_{\mu}$. This is simply a consequence of total energy conservation; whatever energy is input into the system has to either reach dissipation scales by way of the SGS flux or get converted into internal energy through pressure dilatation.

The results of this paper lay the mathematical framework upon which we based previous published work Aluie (2011); Aluie et al. (2012). This enabled us to address basic questions pertaining to the cascade in compressible turbulence where we showed that kinetic energy cascades conservatively despite 
not being in invariant of the dynamics, and that such a cascade process is dominated by interactions between scales of similar size.

Acknowledgements. I thank G. L. Eyink for invaluable discussions and for suggesting the idea of the proof in Proposition 5 instead of a more complicated proof in an earlier version of the manuscript Aluie (2011). I also thank D. Livescu for his input on compressible and variable-density flows, S. S. Girimaji and S. K. Lele for helpful suggestions, and X. Asay-Davis for constructive comments. I wish to acknowledge the useful suggestions of two anonymous referees, and the encouragement of R. Ecke, S. Kurien, S. Li, H. Li, and B. Wingate during this project. This research was performed under the auspices of the U.S. Department of Energy at LANL under Contract No. DE-AC52-06NA25396 and supported by the LANL/LDRD program.

\section{A Viscous effects}

\section{A.1 Constant viscous coefficient}

In general, dynamic viscosity is a spatially varying quantity that is well-described by Sutherland's law or a simpler power law dependence on temperature, $\mu(\mathbf{x}) \sim T(\mathbf{x})^{\alpha}$. Hence, it is constant in isothermal flows or approximately so in flows with a small Prandtl number where thermal conductivity is large enough to homogenize the temperature field at scales $\leq \ell_{\kappa}$. Propositions 1 and 2 below apply to these special cases. In Propositions 3 and 4 , we generalize the proofs to flows with a spatially variable $\mu(\mathbf{x})$.

In the following proposition, we prove rigorously that viscous terms in the balance 10$)$ of large-scale momentum $\bar{\rho}_{\ell} \widetilde{\mathbf{u}}_{\ell}$ are negligible for small viscosity $\mu \rightarrow 0$. Proposition 1 is very similar to that given in Eyink (2007) and Aluie (2009). To avoid additional complications due to boundaries, we consider a domain $\mathbb{T}^{d}=[0,2 \pi)^{d}$ that is periodic.

Proposition 1. For a constant viscosity, $\mu(\mathbf{x})=\mu$, if velocity solutions $\mathbf{u}$ of the compressible NavierStokes equation (1)-(3) over a domain $\mathbb{T}^{d}$ have finite 2 nd-order moments: $\int_{\mathbb{T}^{d}} d \mathbf{x}|\mathbf{u}|^{2}<\infty$, then viscous terms in the large-scale momentum eq. (8) vanish pointwise as $\mu \rightarrow 0$.

Proof of Proposition 1:

Using integration by parts, every viscous term in eq. (8) can be rewritten as,

$$
\mu \partial_{j} \partial_{i} \overline{\mathbf{u}}_{\ell}(\mathbf{x})=\frac{\mu}{\ell^{2}} \int d \mathbf{r}\left(\partial_{j} \partial_{i} G\right)_{\ell}(\mathbf{r}) \mathbf{u}(\mathbf{x}+\mathbf{r}),
$$


where $\left(\partial_{i} G\right)_{\ell}(\mathbf{r})=\ell^{-d} \partial G(\mathbf{r} / \ell) / \partial\left(r_{i} / \ell\right)$. This can be bounded by Hölder's inequality for $1 / p+1 / q=1$,

$$
\begin{aligned}
\left|\mu \partial_{j} \partial_{i} \overline{\mathbf{u}}_{\ell}(\mathbf{x})\right| & \leq \frac{\mu}{\ell^{2}} \int d \mathbf{r}\left|\left(\partial_{j} \partial_{i} G\right)_{\ell}(\mathbf{r}) \mathbf{u}(\mathbf{x}+\mathbf{r})\right| \\
& \leq \frac{\mu}{\ell^{2}} V^{\frac{1}{p}}\left\|\left(\partial_{j} \partial_{i} G\right)_{\ell}\right\|_{p} V^{\frac{1}{q}}\|\mathbf{u}\|_{q}=\frac{\mu}{\ell^{2}}\left(\frac{L_{\mathrm{dom}}}{\ell}\right)^{d\left(1-\frac{1}{p}\right)}\|\mathbf{u}\|_{q}\left(\int d \mathbf{s}\left|\frac{\partial^{2} G(\mathbf{s})}{\partial s_{i} \partial s_{j}}\right|^{p}\right)^{\frac{1}{p}}
\end{aligned}
$$

where $\|\ldots\|_{p}=\left\langle|\ldots|^{p}\right\rangle^{1 / p}$ is the $L_{p}$-norm, $L_{\text {dom }}^{d}=V$ is the domain's volume, and $\mathbf{s}=\mathbf{r} / \ell$ is a dimensionless vector. Since $G(\mathbf{s}) \in C^{\infty}$, its derivatives are uniformly bounded and we have $\left\|\partial_{j} \partial_{i} G\right\|_{p}=$ (const.) $<\infty$ for any $p$, including $p=\infty$. Choosing $p=q=2$, our bound implies that the viscous term $\mu \partial_{j} \partial_{i} \overline{\mathbf{u}}_{\ell} \rightarrow 0$ at every point in space in the limit of vanishing viscosity.

REMARK: We could have obtained a tighter bound in terms of $\delta u(\ell) \equiv \sup _{|\mathbf{r}|<\ell}|\delta \mathbf{u}(\mathbf{x} ; \mathbf{r})|$ by rewriting the viscous term as

$$
\mu \partial_{j} \partial_{i} \overline{\mathbf{u}}_{\ell}(\mathbf{x})=\frac{\mu}{\ell^{2}} \int d \mathbf{r}\left(\partial_{j} \partial_{i} G\right)_{\ell}(\mathbf{r})(\mathbf{u}(\mathbf{x}+\mathbf{r})-\mathbf{u}(\mathbf{x})),
$$

exploiting the fact that $\int d \mathbf{r}\left(\partial_{j} \partial_{i} G\right)_{\ell}(\mathbf{r})=0$ due to the decay of $G(\mathbf{s})$ at $\pm \infty$ or due to periodic boundary conditions (see Eyink Eyink (2005)). Such a bound would then be of the form $O\left(\mu / \ell^{2} \delta u(\ell)\right)$ for kernels $G$ with compact support Eyink (2007). A stronger assumption would then be required, that $\delta u(\ell) \equiv \sup _{|\mathbf{r}|<\ell}|\delta \mathbf{u}(\mathbf{x} ; \mathbf{r})|<\infty$ at $\mathbf{x}$.

The following proposition proves that viscous dissipation of large-scale kinetic energy $\frac{1}{2} \bar{\rho}_{\ell}\left|\widetilde{\mathbf{u}}_{\ell}\right|^{2}$ in eq. 13 becomes negligible at any point $\mathbf{x}$ in the limit of small $\mu$. The assumptions of finite 3rd-order moments for the velocity and density fields, $\left\langle|\mathbf{u}|^{3}\right\rangle\left\langle\infty\right.$ and $\left\langle|\rho|^{3}\right\rangle\langle\infty$, are weak and are expected to hold in any physically realizable flow. The assumption of finite mean specific volume, $\langle 1 / \rho\rangle\langle\infty$, is used to control factors $1 / \bar{\rho}_{\ell}(\mathbf{x})$ in the dissipation terms. Coarse-grained density $\bar{\rho}_{\ell}(\mathbf{x})$, for positive filter kernels $G(\mathbf{r}) \geq 0$, is proportional to the mass in a ball of radius $\ell$ centered around $\mathbf{x}$. The assumption $\langle 1 / \rho\rangle<\infty$ guarantees that mass in a ball of any finite radius $\ell>0$, at any location $\mathbf{x}$, will contain non-zero mass. The assumption still allows for regions with $\rho(\mathbf{x})=0$, but such regions must occupy zero volume (i.e. zero Lebesgue measure). In other words, Proposition 2 does not hold in vacuum pockets of non-zero volume where viscous dissipation is meaningless.

Proposition 2. For a constant $\mu(\mathbf{x})=\mu$, if solutions $(\rho, \mathbf{u})$ of the compressible Navier-Stokes equation (1)-(3) over domain $\mathbb{T}^{d}$ have finite 3rd-order moments: $\int_{\mathbb{T}^{d}} d \mathbf{x}|\rho|^{3}<\infty$ and $\int_{\mathbb{T}^{d}} d \mathbf{x}|\mathbf{u}|^{3}<\infty$, and finite mean specific volume, $\int_{\mathbb{T}^{d}} d \mathbf{x} \rho^{-1}<\infty$, then for positive kernels $G(\mathbf{r}) \geq 0$, viscous terms in the large-scale kinetic energy budget (13) vanish pointwise as $\mu \rightarrow 0$. 
Proof of Proposition 2:

Using the exact identity $\widetilde{\mathbf{u}}=\overline{\mathbf{u}}+\bar{\tau}(\rho, \mathbf{u}) / \bar{\rho}$, we have

$$
\left|\partial_{j} \widetilde{u}_{i}(\mathbf{x})\right| \leq\left|\partial_{j} \bar{u}_{i}\right|+\left|\frac{1}{\bar{\rho}} \partial_{j} \bar{\tau}\left(\rho, u_{i}\right)\right|+\left|\frac{1}{\bar{\rho}^{2}} \bar{\tau}\left(\rho, u_{i}\right) \partial_{j} \bar{\rho}\right| .
$$

The first term $\left|\partial_{j} \bar{u}_{i}\right|$ is bounded by

$$
\begin{aligned}
& \ell^{-1}\|\mathbf{u}\|_{3}(\text { const. })\left\|(\nabla G)_{\ell}\right\|_{2} \\
= & \ell^{-1}\|\mathbf{u}\|_{3} A\left(L_{\mathrm{dom}} / \ell\right)
\end{aligned}
$$

through an argument identical to that in Proposition 1 and using the fact that $\|\mathbf{u}\|_{2} \leq$ (const.) $\|\mathbf{u}\|_{3}$ over a bounded domain. Here, $A\left(L_{\mathrm{dom}} / \ell\right)=($ const. $)\left(L_{\mathrm{dom}} / \ell\right)^{d / 2}\|\nabla G\|_{2}$ is dimensionless.

The second term in 33 can be rewritten as

$$
\begin{gathered}
\frac{1}{\bar{\rho}} \partial_{j} \bar{\tau}\left(\rho, u_{i}\right)=-\frac{1}{\bar{\rho} \ell}\left[\int d \mathbf{r}\left(\partial_{j} G\right)_{\ell}(\mathbf{r}) \rho(\mathbf{x}+\mathbf{r}) u_{i}(\mathbf{x}+\mathbf{r})\right. \\
\left.-\int d \mathbf{r}\left(\partial_{j} G\right)_{\ell}(\mathbf{r}) \rho(\mathbf{x}+\mathbf{r}) \int d \mathbf{r}^{\prime} G_{\ell}\left(\mathbf{r}^{\prime}\right) u_{i}\left(\mathbf{x}+\mathbf{r}^{\prime}\right)-\int d \mathbf{r} G_{\ell}(\mathbf{r}) \rho(\mathbf{x}+\mathbf{r}) \int d \mathbf{r}^{\prime}\left(\partial_{j} G\right)_{\ell}\left(\mathbf{r}^{\prime}\right) u_{i}\left(\mathbf{x}+\mathbf{r}^{\prime}\right)\right],
\end{gathered}
$$

using integration by parts. Employing the 3-3-3 Hölder's inequality, this expression is bounded by

$$
\begin{aligned}
& (\text { const. }) \ell^{-1} \frac{1}{\bar{\rho}}\|\rho\|_{3}\|\mathbf{u}\|_{3}\left(1+2\|1\|_{3}^{2}\left\|G_{\ell}\right\|_{3}\right)\left\|(\nabla G)_{\ell}\right\|_{3} \\
= & \ell^{-1} \frac{1}{\bar{\rho}}\|\rho\|_{3}\|\mathbf{u}\|_{3} B\left(L_{\mathrm{dom}} / \ell\right)
\end{aligned}
$$

where $B\left(L_{\mathrm{dom}} / \ell\right)=\left((\right.$ const. $)\left(\frac{L \mathrm{dom}}{\ell}\right)^{\frac{2 d}{3}}+($ const. $\left.)\left(\frac{L \mathrm{dom}}{\ell}\right)^{\frac{4 d}{3}}\right)$ is dimensionless.

The third term in 33 can be rewritten as

$$
\begin{gathered}
\frac{1}{\bar{\rho}^{2}} \bar{\tau}\left(\rho, u_{i}\right) \partial_{j} \bar{\rho}=-\bar{\rho}^{-2} \ell^{-1} \int d \mathbf{r}\left(\partial_{j} G\right)_{\ell}(\mathbf{r}) \rho(\mathbf{x}+\mathbf{r}) \\
\times\left[\int d \mathbf{r} G_{\ell}(\mathbf{r}) \rho(\mathbf{x}+\mathbf{r}) u_{i}(\mathbf{x}+\mathbf{r})-\int d \mathbf{r} G_{\ell}(\mathbf{r}) \rho(\mathbf{x}+\mathbf{r}) \int d \mathbf{r}^{\prime} G_{\ell}\left(\mathbf{r}^{\prime}\right) u_{i}\left(\mathbf{x}+\mathbf{r}^{\prime}\right)\right] .
\end{gathered}
$$

Using Hölder's inequality, this is bounded by

$$
\begin{aligned}
& (\text { const. }) \ell^{-1} \frac{1}{\bar{\rho}^{2}}\|\rho\|_{3}^{2}\|\mathbf{u}\|_{3}\left(1+\|1\|_{3}\left\|G_{\ell}\right\|_{3}\right)\left\|(\nabla G)_{\ell}\right\|_{3}\|1\|_{3}\left\|G_{\ell}\right\|_{3} \\
= & \ell^{-1} \frac{1}{\bar{\rho}^{2}}\|\rho\|_{3}^{2}\|\mathbf{u}\|_{3} C\left(L_{\mathrm{dom}} / \ell\right)
\end{aligned}
$$

where $C(L \mathrm{dom} / \ell)=\left((\right.$ const. $)\left(\frac{L \mathrm{dom}}{\ell}\right)^{\frac{4 d}{3}}+($ const. $\left.)\left(\frac{L \mathrm{dom}}{\ell}\right)^{2 d}\right)$ is dimensionless.

Finally, each of the viscous terms is bounded by

$$
\mu|\nabla \widetilde{\mathbf{u}} \nabla \overline{\mathbf{u}}| \leq \frac{\mu}{\ell^{2}}\|\mathbf{u}\|_{3}^{2}\left[A\left(L_{\mathrm{dom}} / \ell\right)+B(L \mathrm{dom} / \ell) \frac{\|\rho\|_{3}}{\bar{\rho}}+C\left(L_{\mathrm{dom}} / \ell\right) \frac{\|\rho\|_{3}^{2}}{\bar{\rho}^{2}}\right] A\left(L_{\mathrm{dom}} / \ell\right) .
$$


Factors $1 / \bar{\rho}_{\ell}(\mathbf{x})$ in the above expression are finite because $1 / \rho$ is a convex function of density over $\rho \in[0, \infty)$. When $G(\mathbf{r}) \geq 0$, coarse-graining is an averaging operation and we can use Jensen's inequality to obtain

$$
1 / \bar{\rho}_{\ell}(\mathbf{x}) \leq(\overline{1 / \rho})_{\ell}(\mathbf{x}) \leq\left\|G_{\ell}\right\|_{p}\left\|\rho^{-1}\right\|_{q}=\left\|\rho^{-1}\right\|_{q}\left(\frac{L \mathrm{dom}}{\ell}\right)^{d\left(1-\frac{1}{p}\right)}\|G\|_{p}
$$

where we used Hölder's inequality to obtaining the second inequality with $1 / p+1 / q=1$. For $p=\infty$ and $q=1$, we have that $1 / \bar{\rho}_{\ell}(\mathbf{x})<\infty$ for any fixed $\ell>0$ under the assumption $\langle 1 / \rho\rangle<\infty$.

Hence, for any fixed $\ell>0$, a viscous term $\mu \nabla \widetilde{\mathbf{u}} \nabla \overline{\mathbf{u}} \rightarrow 0$ at every point in space in the limit of vanishing viscosity.

\section{A.2 Spatially varying viscous coefficient}

We now extend the previous two propositions to the case when $\mu(\mathbf{x})$ varies in space. In the proofs below, we require that spatial gradients of viscosity are bounded (in a root-mean-square sense) and vanish when $\mu_{\mathrm{rms}} \rightarrow 0$. The assumption can be checked directly from a series of direct numerical simulations at increasingly higher resolution. Empirical support (or refutation) for the assumption can also be obtained by measuring the spectrum of $\mu(\mathbf{x})$, namely $E^{\mu}(k)=\sum_{k-1<|\mathbf{k}| \leq k}|\widehat{\mu}(\mathbf{k})|^{2}$ or, alternatively, by measuring the temperature spectrum based on a relation $\mu=\mu(T)$.

A spectrum $E^{\mu}(k)$ that decays faster than $k^{-1 / 3}$ would support to our assumption on $\nabla \mu$ based on the following physical reasoning. $\mu(T(\mathbf{x}))$ varies on scales $\geq \ell_{\kappa}$, the temperature dissipation scale. If such variations scale as $\delta \mu(\ell) \sim \mu(\ell / L)^{h_{\mu}}$ for $\ell \gg \ell_{\kappa}$, corresponding to a spectrum $E^{\mu}(k) \sim k^{-2 h_{\mu}-1}$, then gradients scale as

$$
\nabla \mu \sim \delta \mu(\ell) / \ell \sim \mu(\ell / L)^{h_{\mu}-1}
$$

If $h_{\mu} \geq 1$, the field $\mu(\mathbf{x})$ is smooth enough to guarantee that gradients have little contribution from small scales and $\nabla \mu$ will vanish as $\mu \rightarrow 0$. This is also true if $E^{\mu}(k)$ decays faster than any power law (e.g. exponentially). On the other hand, if $h_{\mu}<1$, most of the contribution to $\nabla \mu$ comes from the smallest scales in the temperature field and we have

$$
\nabla \mu \sim \mu\left(\ell_{\kappa} / L\right)^{h_{\mu}-1}
$$

Suppose the temperature dissipation scale, $\ell_{\kappa}$, can be determined from the Obukhov-Corrsin-Batchelor 
phenomenology of passive scalars Obukhov (1949); Corrsin (1951); Batchelor (1959):

$$
\begin{array}{ll}
\ell_{\kappa}=\left(\frac{\kappa^{3}}{\langle\rho\rangle^{2} \epsilon_{\mathrm{net}} c_{p}^{3}}\right)^{\frac{1}{4}}, & \text { for } \operatorname{Pr} \leq 1 \\
\ell_{\kappa}=\left(\frac{\kappa^{2} \mu}{\langle\rho\rangle^{2} \epsilon_{\mathrm{net}} c_{p}^{2}}\right)^{\frac{1}{4}}=\ell_{\mu} \operatorname{Pr}^{-\frac{1}{2}}, & \text { for } \operatorname{Pr}>1,
\end{array}
$$

where $\epsilon_{\text {net }}=\langle\rho \mathbf{u} \cdot \mathbf{F}+P \boldsymbol{\nabla} \cdot \mathbf{u}\rangle$ is the net kinetic energy reaching the dissipation scales (see eq. (10) in Aluie Aluie (2011) or eq. (27) above). The Prandtl number is $\operatorname{Pr}=c_{p} \mu / \kappa$, where $c_{p}$ is specific heat at constant pressure. The second equality in eq. (38) assumes that the kinetic dissipation scale is determined from a Kolmogorov-type estimate $\ell_{\mu}=\left(\mu^{3} /\langle\rho\rangle^{2} \epsilon_{\text {net }}\right)^{1 / 4}$.

It follows from relations $(37), 38)$ that viscosity gradients vanish when $\mu \rightarrow 0$ for a fixed $\operatorname{Pr}$ (i.e. in the limit of small $\mu$ and small $\kappa$ while keeping their ratio constant),

$$
\begin{aligned}
& \nabla \mu \sim \mu\left(\ell_{\kappa} / L\right)^{h_{\mu}-1} \sim \mu^{\frac{3 h_{\mu}+1}{4}} \operatorname{Pr}^{\frac{3}{4}\left(1-h_{\mu}\right)}, \quad \text { for } \operatorname{Pr} \leq 1, \\
& \nabla \mu \sim \mu\left(\ell_{\kappa} / L\right)^{h_{\mu}-1} \sim \mu^{\frac{3 h_{\mu}+1}{4}} \operatorname{Pr} r^{\frac{1-h_{\mu}}{2}}, \quad \text { for } \operatorname{Pr}>1,
\end{aligned}
$$

under the very weak condition that $h_{\mu}>-1 / 3$. If, on the other hand, we consider the limit $\mu \rightarrow 0$ for a fixed $\kappa$ (i.e. in the limit of small $\mu$ and small $\operatorname{Pr}$ ), then $\nabla \mu \sim \mu$ vanishes for any value of the scaling exponent $h_{\mu}$, as one would expect physically for flows in the $\operatorname{Pr} \rightarrow 0$ limit where thermal conductivity becomes large enough to homogenize the temperature field.

Measuring $E^{\mu}(k)$ from direct numerical simulations is simple although we are not aware of any such result reported in the literature. We note that a decay rate of $E^{\mu}(k)<$ (const.) $k^{-1 / 3}$ is a weak condition on any reasonable field. Alternatively, since viscosity is related to temperature through Sutherland's law or a simpler power law, $\mu(\mathbf{x})=\mu_{0}\left(T(\mathbf{x}) / T_{0}\right)^{\alpha}$, one can infer the spectrum $E^{\mu}(k)$ from that of temperature, $E^{T}(k)$. This follows from $\mu(T)$ being a Lipschitz function of temperature and $T(\mathbf{x}) \geq$ $T_{\min }>0$ :

$$
\delta \mu(\ell)=\mu(x+\ell)-\mu(x)=\frac{\mu_{0}}{T_{0}^{\alpha}} \alpha T_{\mathrm{cst}}^{\alpha-1}[T(x+\ell)-T(x)]=(\text { const. }) \mu_{0}\left(\frac{\ell}{L}\right)^{h_{T}},
$$

where we used the mean value theorem in the second equality with $T_{\mathrm{cst}} \in(T(x), T(x+\ell))$, and $\delta T(\ell)=$ (const.) $T_{\mathrm{rms}}(\ell / L)^{h_{T}}$ in the last equality. Hence, fluctuations in $\mu$ have the same scaling exponent as those of the temperature field, $h_{\mu}=h_{T}$. We note that the physical arguments presented thus far in this subsection A.2 are not rigorous but serve to justify the assumption on $\nabla \mu$ used in the rigorous proofs of Propositions 3 and 4 
Proposition 3. If velocity solutions $\mathbf{u}$ of the compressible Navier-Stokes equation (1)-(3) over a domain $\mathbb{T}^{d}$ have finite 2 nd-order moments: $\int_{\mathbb{T}^{d}} d \mathbf{x}|\mathbf{u}|^{2}<\infty$, and viscous gradients are bounded as follows:

$$
\left\langle|\nabla \mu(\mathbf{x})|^{2}\right\rangle^{\frac{1}{2}} \leq A \frac{\mu_{\mathrm{rms}}}{L}\left(\frac{\mu_{\mathrm{rms}}}{M}\right)^{\frac{3}{4}\left(h_{\mu}-1\right)},
$$

where $\mu_{\mathrm{rms}}=\left\langle\mu^{2}\right\rangle^{1 / 2}, \beta=3 / 4\left(h_{\mu}-1\right)>-1$ and constants $A, L$, and $M$, then viscous terms in the large-scale momentum eq. (8) vanish pointwise as $\mu_{\mathrm{rms}} \rightarrow 0$.

Proof of Proposition 3:

By integration by parts, any of the viscous terms in eq. (8) can be rewritten as,

$$
\begin{aligned}
\partial_{j}\left(\overline{\mu \partial_{i} \mathbf{u}}\right)_{\ell}(\mathbf{x}) & =\frac{1}{\ell^{2}} \int d \mathbf{r}\left(\partial_{i} \partial_{j} G\right)_{\ell}(\mathbf{r}) \mu(\mathbf{x}+\mathbf{r}) \mathbf{u}(\mathbf{x}+\mathbf{r}) \\
& +\frac{1}{\ell} \int d \mathbf{r}\left(\partial_{j} G\right)_{\ell}(\mathbf{r}) \mathbf{u}(\mathbf{x}+\mathbf{r}) \partial_{i} \mu(\mathbf{x}+\mathbf{r}) .
\end{aligned}
$$

This can be bounded by

$$
\begin{aligned}
\left|\partial_{j}\left(\overline{\mu \partial_{i} \mathbf{u}}\right)_{\ell}(\mathbf{x})\right| & \leq \frac{1}{\ell^{2}}\left\|\left(\partial_{i} \partial_{j} G\right)_{\ell}\right\|_{\infty}\|\mu\|_{2}\|\mathbf{u}\|_{2}+\frac{1}{\ell}\left\|\left(\partial_{j} G\right)_{\ell}\right\|_{\infty}\|\mathbf{u}\|_{2}\|\nabla \mu\|_{2} \\
& \leq \frac{\mu_{\mathrm{rms}}}{\ell^{2}}\|\mathbf{u}\|_{2}\left(\frac{L_{\mathrm{dom}}}{\ell}\right)^{d}\left\|\partial_{i} \partial_{j} G\right\|_{\infty}+\frac{\mu_{\mathrm{rms}}^{1+\beta}}{\ell L M^{\beta}} A\|\mathbf{u}\|_{2}\left(\frac{L_{\mathrm{dom}}}{\ell}\right)^{d}\left\|\partial_{j} G\right\|_{\infty},
\end{aligned}
$$

which, for any fixed $\ell>0$, vanishes as $\mu_{\mathrm{rms}} \rightarrow 0$.

REMARK: In our choice of $\mu_{\mathrm{rms}}$ when deriving the above bound, we imagined a field $\mu(\mathbf{x})$ that is statistically homogeneous. If $\mu(\mathbf{x})$ in a region of interest is significantly different (in a statistical sense) from the rest of the domain, other more optimal bounds can be derived using other moments of $\mu(\mathbf{x})$ such as $\|\mu\|_{L_{\text {loc }}^{2}}$ or $\max _{|\mathbf{x}+\mathbf{r}|<\ell} \mu(\mathbf{x}+\mathbf{r})$.

Proposition 4. If solutions $(\rho, \mathbf{u})$ of the compressible Navier-Stokes equation (1)-(3) over domain $\mathbb{T}^{d}$ have finite 3rd-order moments: $\int_{\mathbb{T}^{d}} d \mathbf{x}|\rho|^{3}<\infty$ and $\int_{\mathbb{T}^{d}} d \mathbf{x}|\mathbf{u}|^{3}<\infty$, a finite mean specific volume, $\int_{\mathbb{T}^{d}} d \mathbf{x} \rho^{-1}<\infty$, and viscous gradients are bounded as follows:

$$
\left\langle|\nabla \mu(\mathbf{x})|^{2}\right\rangle^{\frac{1}{2}} \leq A \frac{\mu_{\mathrm{rms}}}{L}\left(\frac{\mu_{\mathrm{rms}}}{M}\right)^{\frac{3}{4}\left(h_{\mu}-1\right)},
$$

where $\left.\mu_{\mathrm{rms}}=\left\langle\mu^{2}\right\rangle^{1 / 2}, \beta=3 / 4\left(h_{\mu}-1\right)\right\rangle-1$ and constants $A, L$, and $M$, then for positive kernels $G(\mathbf{r}) \geq 0$, viscous terms in the large-scale kinetic energy budget 13 vanish pointwise as $\mu_{\mathrm{rms}} \rightarrow 0$.

Proof of Proposition 4:

As in Proposition 2 eqs. (33)- 36, we have the following bound on $\nabla \widetilde{\mathbf{u}}$ :

$$
|\nabla \widetilde{\mathbf{u}}| \leq \frac{1}{\ell}\|\mathbf{u}\|_{3}\left[A\left(L_{\mathrm{dom}} / \ell\right)+B\left(L_{\mathrm{dom}} / \ell\right) \frac{\|\rho\|_{3}}{\bar{\rho}}+C\left(L_{\mathrm{dom}} / \ell\right) \frac{\|\rho\|_{3}^{2}}{\bar{\rho}^{2}}\right] .
$$


In a derivation similar to that in Proposition 3, we obtain the following bound

$$
\begin{aligned}
|\overline{\mu \boldsymbol{\nabla u}}(\mathbf{x})| & \leq \frac{1}{\ell}\left\|(\nabla G)_{\ell}\right\|_{\infty}\|\mu\|_{2}\|\mathbf{u}\|_{2}+\left\|G_{\ell}\right\|_{\infty}\|\mathbf{u}\|_{2}\|\nabla \mu\|_{2} \\
& \leq \text { (const. }) \frac{\mu_{\mathrm{rms}}}{\ell}\|\mathbf{u}\|_{3}\left(\frac{L_{\mathrm{dom}}}{\ell}\right)^{d}\|\nabla G\|_{\infty}+\text { (const.) } \frac{\mu_{\mathrm{rms}}^{1+\beta}}{L M^{\beta}}\|\mathbf{u}\|_{3}\left(\frac{L_{\mathrm{dom}}}{\ell}\right)^{d}\|G\|_{\infty},
\end{aligned}
$$

Hence, we have

$$
\begin{aligned}
|\nabla \widetilde{\mathbf{u}} \overline{\mu \nabla \mathbf{u}}(\mathbf{x})| \leq \frac{\mu_{\mathrm{rms}}}{\ell^{2}}\|\mathbf{u}\|_{3}^{2} & {\left[A\left(L_{\mathrm{dom}} / \ell\right)+B\left(L_{\mathrm{dom}} / \ell\right) \frac{\|\rho\|_{3}}{\bar{\rho}}+C(L \mathrm{dom} / \ell) \frac{\|\rho\|_{3}^{2}}{\bar{\rho}^{2}}\right] } \\
\times & {\left[(\text { const. })+(\text { const. }) \frac{\ell}{L} \frac{\mu_{\mathrm{rms}}^{\beta}}{M^{\beta}}\right]\left(\frac{L_{\mathrm{dom}}}{\ell}\right)^{d} }
\end{aligned}
$$

As discussed in Proposition 2 , factors $1 / \bar{\rho}_{\ell}(\mathbf{x})$ are bounded by

$$
1 / \bar{\rho}_{\ell}(\mathbf{x}) \leq(\overline{1 / \rho})_{\ell}(\mathbf{x}) \leq\left\|G_{\ell}\right\|_{p}\left\|\rho^{-1}\right\|_{q}=\left\|\rho^{-1}\right\|_{q}\left(\frac{L_{\mathrm{dom}}}{\ell}\right)^{d\left(1-\frac{1}{p}\right)}\|G\|_{p} .
$$

It follows that for any fixed $\ell>0$, viscous dissipation in eq. (13), $D_{\ell}(\mathbf{x}) \rightarrow 0$, at every $\mathbf{x}$ in the limit $\mu_{\mathrm{rms}} \rightarrow 0$.

\section{B Kinetic energy injection}

The following proposition proves that mean kinetic energy injection is localized to the largest scales $\gtrsim L \sim K_{0}^{-1}$ when stirring with an external acceleration field. To this end, we need to show that

$$
\left\langle\epsilon_{\ell}^{i n j}\right\rangle=\left\langle\bar{\rho} \widetilde{u}_{i} \widetilde{F}_{i}\right\rangle \longrightarrow\left\langle\left(\rho u_{i}\right)^{<K_{0}} F_{i}^{<K_{0}}\right\rangle,
$$

as $K_{0} \ell \rightarrow 0$. In our proof, we will assume that the external acceleration field has only small wavenumber modes $<K_{0}$ as is traditionally done in numerical simulations (e.g. Kida and Orszag (1990); Schmidt et al. (2009); Federrath et al. (2010); Kritsuk et al. (2007)) such that $\mathbf{F}(\mathbf{x})=\mathbf{F}^{<K_{0}}(\mathbf{x})$ for some $K_{0}>0$ (the case of $K_{0}=0$ was discussed in section 5.2). We will also assume that density fluctuations decay fast enough (or at least grow slowly) at small scales such that for some constant $A_{2},\|\delta \rho(\mathbf{r})\|_{2} \leq$ $\rho_{r m s} A_{2}\left(r K_{0}\right)^{\sigma_{2}^{\rho}}$ with $\sigma_{2}^{\rho}>-1$ as $r \rightarrow 0$. This condition corresponds to a density spectrum $E^{\rho}(k) \equiv$ $\sum_{k-0.5<|\mathbf{k}|<k+0.5}|\hat{\rho}(\mathbf{k})|^{2} \leq$ (const.) $k^{-\alpha}$ with $\alpha=2 \sigma_{2}^{\rho}+1>-1$, which is readily satisfied for any density field with finite $\rho_{r m s}$. Such an assumption is only an upper bound and does not even require that the density spectrum be continuous. We also assume that $\left\langle 1 / \rho^{4}\right\rangle<\infty$, which is slightly stronger that an assumption of finite mean specific volume used in Proposition 2. Regarding the filtering kernel, we assume that it decays fast enough with $r \rightarrow \infty$ such that $\int d \mathbf{r}|G(\mathbf{r})||\mathbf{r}|^{\beta}=($ const. $)<\infty$ for $\beta=1, \sigma_{2}^{\rho}$, and $1+\sigma_{2}^{\rho}$, which hold for any reasonable kernel with finite spread. 
Proposition 5. If solutions $(\rho, \mathbf{u})$ of the compressible Navier-Stokes equation (1)-(3), stirred with an acceleration $\mathbf{F}(\mathbf{x})=\mathbf{F}^{<K_{0}}(\mathbf{x})$ in a periodic domain $\mathbb{T}^{d}=[0,2 \pi)^{d}$, satisfy $\|\rho \mathbf{u}\|_{4}<\infty,\|1 / \rho\|_{4}<\infty$, and $\|\delta \rho(\mathbf{r})\|_{2} \leq \rho_{r m s} A_{2}\left(r K_{0}\right)^{\sigma_{2}^{\rho}}$, then

$$
\begin{aligned}
& \left|\left\langle\epsilon_{\ell}^{i n j}-\left(\rho u_{i}\right)^{<K_{0}} F_{i}^{<K_{0}}\right\rangle\right| \\
& \quad \leq \text { (const.) } \rho_{r m s}\left\|\rho^{-1}\right\|_{4}\|\rho \mathbf{u}\|_{4}\|\mathbf{F}\|_{1}\left(\ell K_{0}\right)^{1+\sigma_{2}^{\rho}}+(\text { const. })\|\rho \mathbf{u}\|_{1}\|\mathbf{F}\|_{1}\left(\ell K_{0}\right)^{2}
\end{aligned}
$$

when $G(\mathbf{r}) \geq 0$ and $\int d \mathbf{r}|G(\mathbf{r})||\mathbf{r}|^{\beta}=$ (const.) $<\infty$ for $\beta=1, \sigma_{2}^{\rho}$, and $1+\sigma_{2}^{\rho}$. The upper bound 40] vanishes in the limit $\ell K_{0} \rightarrow 0$ for any $\sigma_{2}^{\rho}>-1$.

\section{Proof of Proposition 5:}

Rewriting the kinetic energy injection as

$$
\epsilon_{\ell}^{i n j} \equiv \bar{\rho} \widetilde{u}_{i} \widetilde{F}_{i}=\frac{\overline{\rho u_{i}}}{\bar{\rho}} \overline{\rho F_{i}}=\frac{\overline{\rho u_{i}}}{\bar{\rho}}\left[\bar{\rho} \bar{F}_{i}+\bar{\tau}\left(\rho, F_{i}\right)\right]
$$

we have

$$
\begin{aligned}
& \left\langle\epsilon_{\ell}^{i n j}-\left(\rho u_{i}\right)^{<K_{0}} F_{i}^{<K_{0}}\right\rangle \\
= & \left\langle\overline{\rho u_{i}} \bar{F}_{i}-\left(\overline{\rho u_{i}}\right)^{<K_{0}}\left(\bar{F}_{i}\right)^{<K_{0}}\right\rangle+\left\langle\frac{\overline{\rho u_{i}}}{\bar{\rho}} \bar{\tau}\left(\rho, F_{i}\right)\right\rangle-\left\langle\bar{\tau}\left(\left(\rho u_{i}\right)^{<K_{0}}, F_{i}^{<K_{0}}\right)\right\rangle,
\end{aligned}
$$

where we used $\int d \mathbf{x} f(\mathbf{x})=\int d \mathbf{x} \bar{f}(\mathbf{x})$ in the last term. Due to commutativity and associativity of convolutions, $\overline{\left(\mathbf{F}^{<K_{0}}\right)}=(\overline{\mathbf{F}})^{<K_{0}}$, the orthogonality of Fourier modes, $\left\langle\overline{\rho u_{i}}\left(\bar{F}_{i}\right)^{<K_{0}}\right\rangle=\left\langle\left(\overline{\rho u_{i}}\right)^{<K_{0}} \bar{F}_{i}^{<K_{0}}\right\rangle$, yields

$$
\begin{aligned}
\left|\left\langle\epsilon_{\ell}^{i n j}-\left(\rho u_{i}\right)^{<K_{0}} F_{i}^{<K_{0}}\right\rangle\right|= & \left|\left\langle\frac{\overline{\rho u_{i}}}{\bar{\rho}} \bar{\tau}\left(\rho, F_{i}\right)\right\rangle-\left\langle\bar{\tau}\left(\left(\rho u_{i}\right)^{<K_{0}}, F_{i}^{<K_{0}}\right)\right\rangle\right| \\
\leq & \left\|\frac{\overline{\rho u_{i}}}{\bar{\rho}} \bar{\tau}\left(\rho, F_{i}\right)\right\|_{1}+\left\|\bar{\tau}\left(\left(\rho u_{i}\right)^{<K_{0}}, F_{i}^{<K_{0}}\right)\right\|_{1} \\
\leq & \left\|\frac{1}{\bar{\rho}}\right\|_{4}\|\rho \mathbf{u}\|_{4}\|\bar{\tau}(\rho, \mathbf{F})\|_{2} \int d \mathbf{r}\left|G_{\ell}(\mathbf{r})\right| \\
& +\left\|\bar{\tau}\left(\left(\rho u_{i}\right)^{<K_{0}}, F_{i}^{<K_{0}}\right)\right\|_{1}
\end{aligned}
$$

We have $\int d \mathbf{r}\left|G_{\ell}(\mathbf{r})\right|=1$ when $G(\mathbf{r}) \geq 0$. Furthermore, observe that $1 / \rho$ is a convex function of density over $\rho \in[0, \infty)$ such that when $G(\mathbf{r}) \geq 0$, coarse-graining is an averaging operation and we can use Jensen's inequality to obtain $1 / \bar{\rho}_{\ell} \leq(\overline{1 / \rho})_{\ell}$. It follows from 48 that

$$
\begin{aligned}
\mid\left\langle\epsilon_{\ell}^{i n j}\right. & \left.-\left(\rho u_{i}\right)^{<K_{0}} F_{i}^{<K_{0}}\right\rangle \mid \\
& \leq\left\|\frac{1}{\rho}\right\|_{4}\|\rho \mathbf{u}\|_{4}\|\bar{\tau}(\rho, \mathbf{F})\|_{2}+\left\|\bar{\tau}\left(\left(\rho u_{i}\right)^{<K_{0}}, F_{i}^{<K_{0}}\right)\right\|_{1} .
\end{aligned}
$$

It is straightforward to verify the following identity due to Constantin et al. (1994); Eyink (1995),

$$
\begin{aligned}
\bar{\tau}(f, g)(\mathbf{x})= & \int d \mathbf{r} G_{\ell}(\mathbf{r}) \delta f(\mathbf{r} ; \mathbf{x}) \delta g(\mathbf{r} ; \mathbf{x}) \\
& -\int d \mathbf{r}_{1} G_{\ell}\left(\mathbf{r}_{1}\right) \delta f\left(\mathbf{r}_{1} ; \mathbf{x}\right) \int d \mathbf{r}_{2} G_{\ell}\left(\mathbf{r}_{2}\right) \delta g\left(\mathbf{r}_{2} ; \mathbf{x}\right),
\end{aligned}
$$


where $\delta f(\mathbf{r} ; \mathbf{x})=f(\mathbf{x}+\mathbf{r})-f(\mathbf{x})$. Identity 43 allows us to derive the following upper bound:

$$
\begin{aligned}
\|\bar{\tau}(\rho, \mathbf{F})(\mathbf{x})\|_{2} \leq & \int d \mathbf{r}\left|G_{\ell}(\mathbf{r})\right|\|\delta \rho(\mathbf{r} ; \mathbf{x})\|_{2}\|\delta \mathbf{F}(\mathbf{r} ; \mathbf{x})\|_{\infty} \\
& +\int d \mathbf{r}_{1}\left|G_{\ell}\left(\mathbf{r}_{1}\right)\right|\left\|\delta \rho\left(\mathbf{r}_{1} ; \mathbf{x}\right)\right\|_{2} \int d \mathbf{r}_{2}\left|G_{\ell}\left(\mathbf{r}_{2}\right)\right|\left\|\delta \mathbf{F}\left(\mathbf{r}_{2} ; \mathbf{x}\right)\right\|_{\infty} \\
\leq & (\text { const. }) \int d \mathbf{r}\left|G_{\ell}(\mathbf{r})\right|\left(|\mathbf{r}| K_{0}\right)^{\sigma_{2}^{\rho}+1} \\
& +(\text { const. }) \int d \mathbf{r}_{1}\left|G_{\ell}\left(\mathbf{r}_{1}\right)\right|\left(\left|\mathbf{r}_{1}\right| K_{0}\right)^{\sigma_{2}^{\rho}} \int d \mathbf{r}_{2}\left|G_{\ell}\left(\mathbf{r}_{2}\right)\right|\left(\left|\mathbf{r}_{2}\right| K_{0}\right) \\
= & \left(\ell K_{0}\right)^{\sigma_{2}^{\rho}+1}\left\{(\text { const. }) \int d \mathbf{r}|G(\mathbf{r})||\mathbf{r}|^{\sigma_{2}^{\rho}+1}\right. \\
& \left.+(\text { const. }) \int d \mathbf{r}_{1}\left|G\left(\mathbf{r}_{1}\right)\right|\left|\mathbf{r}_{1}\right|^{\sigma_{2}^{\rho}} \int d \mathbf{r}_{2}\left|G\left(\mathbf{r}_{2}\right)\right|\left|\mathbf{r}_{2}\right|\right\} \\
= & O\left(\left(\ell K_{0}\right)^{\sigma_{2}^{\rho}+1}\right),
\end{aligned}
$$

where we used $\mathbf{F}(\mathbf{x})=\mathbf{F}^{<K_{0}}(\mathbf{x})$ to infer the uniform Lipschitz condition, $|\delta \mathbf{F}(\mathbf{r} ; \mathbf{x})| \leq$ (const.) $\|\mathbf{F}\|_{1} K_{0}|\mathbf{r}|$. Using identity (43), we also have

$$
\left\|\bar{\tau}\left(\left(\rho u_{i}\right)^{<K_{0}}, F_{i}^{<K_{0}}\right)\right\|_{1}=O\left(\left(\ell K_{0}\right)^{2}\right),
$$

due to steps similar to those leading to (44). Result 40, follows from bounds 42 , (44), and 45].

The following proposition proves that mean kinetic energy injection is localized to the largest scales $\gtrsim L \sim K_{0}^{-1}$ when stirring with an external forcing field. To this end, we need to show that

$$
\left\langle\epsilon_{\ell}^{i n j}\right\rangle=\left\langle\widetilde{u}_{i} \overline{\mathcal{F}}_{i}\right\rangle \longrightarrow\left\langle u_{i}^{<K_{0}} \mathcal{F}_{i}^{<K_{0}}\right\rangle
$$

as $K_{0} \ell \rightarrow 0$. The bound below is weaker than that obtained in Proposition 5 because $\sigma_{4}^{u}<1$ in a turbulent flow. For example, $\sigma_{4}^{u}=1 / 3$ in Kolmogorov's 1941 theory. This suggest that the rate of convergence is slower than that in Proposition 5 , and that kinetic energy injection is less localized when stirring with $\mathcal{F}^{<K_{0}}$ compared to when stirring with $\rho \mathbf{F}^{<K_{0}}$. This is certainly true when $K_{0}=0$, in which case stirring with $\rho \mathbf{F}_{\text {const }}$ injects energy only at the largest scale, $L_{\text {dom }}$, as discussed in section 5.2 above. On the other hand stirring with $\mathcal{F}_{\text {const }}$ yields an energy injection $\left\langle\epsilon_{\ell}^{i n j}\right\rangle=\mathcal{F}_{\text {const }} \cdot\left\langle\widetilde{\mathbf{u}}_{\ell}\right\rangle=$ $\mathcal{F}_{\text {const }} \cdot\left\langle\bar{\tau}_{\ell}(\rho, \mathbf{u}) / \bar{\rho}_{\ell}\right\rangle$, which in general is non-zero at scales $<L_{\text {dom }}$.

Proposition 6. If solutions ( $\rho, \mathbf{u})$ of the compressible Navier-Stokes equation (1)-(3), stirred with a force $\mathcal{F}(\mathbf{x})=\mathcal{F}^{<K_{0}}(\mathbf{x})$ in a periodic domain $\mathbb{T}^{d}=[0,2 \pi)^{d}$, satisfy $\|1 / \rho\|_{4}<\infty,\|\delta \rho(\mathbf{r})\|_{4} \leq \rho_{r m s} A_{4}\left(r K_{0}\right)^{\sigma_{4}^{\rho}}$, and $\|\delta \mathbf{u}(\mathbf{r})\|_{4} \leq u_{r m s} B_{4}\left(r K_{0}\right)^{\sigma_{4}^{u}}$, then

$$
\begin{aligned}
& \left|\left\langle\epsilon_{\ell}^{i n j}-u_{i}^{<K_{0}} \mathcal{F}_{i}^{<K_{0}}\right\rangle\right| \\
& \quad \leq \text { (const. }) \rho_{r m s} u_{r m s}\left\|\rho^{-1}\right\|_{4}\|\mathcal{F}\|_{4}\left(\ell K_{0}\right)^{\sigma_{4}^{\rho}+\sigma_{4}^{u}}+(\text { const. })\|\mathbf{u}\|_{1}\|\mathcal{F}\|_{1}\left(\ell K_{0}\right)^{2}
\end{aligned}
$$


when $G(\mathbf{r}) \geq 0$ and $\int d \mathbf{r}|G(\mathbf{r})||\mathbf{r}|^{\beta}=$ (const.) $<\infty$ for $\beta=\sigma_{4}^{\rho}$, $\sigma_{4}^{u}$, and $\sigma_{4}^{\rho}+\sigma_{4}^{u}$. The upper bound (47) vanishes in the limit $\ell K_{0} \rightarrow 0$ for any $\sigma_{4}^{\rho}+\sigma_{4}^{u}>0$.

Proof of Proposition 6:

Rewriting the kinetic energy injection as

$$
\epsilon_{\ell}^{i n j} \equiv \widetilde{u}_{i} \overline{\mathcal{F}}_{i}=\frac{\overline{\rho u_{i}}}{\bar{\rho}} \overline{\mathcal{F}}_{i}=\left[\frac{\bar{\rho} \bar{u}_{i}}{\bar{\rho}}+\frac{\bar{\tau}\left(\rho, u_{i}\right)}{\bar{\rho}}\right] \overline{\mathcal{F}}_{i},
$$

we have

$$
\begin{aligned}
& \left\langle\epsilon_{\ell}^{i n j}-u_{i}^{<K_{0}} \mathcal{F}_{i}^{<K_{0}}\right\rangle \\
= & \left\langle\bar{u}_{i} \overline{\mathcal{F}}_{i}-\left(\bar{u}_{i}\right)^{<K_{0}}\left(\overline{\mathcal{F}}_{i}\right)^{<K_{0}}\right\rangle+\left\langle\frac{\bar{\tau}\left(\rho, u_{i}\right)}{\bar{\rho}} \overline{\mathcal{F}}_{i}\right\rangle-\left\langle\bar{\tau}\left(u_{i}^{<K_{0}}, \mathcal{F}_{i}^{<K_{0}}\right)\right\rangle,
\end{aligned}
$$

where we used $\int d \mathbf{x} f(\mathbf{x})=\int d \mathbf{x} \bar{f}(\mathbf{x})$ in the last term. Orthogonality of Fourier modes, $\left\langle\bar{u}_{i}\left(\overline{\mathcal{F}}_{i}\right)^{<K_{0}}\right\rangle=$ $\left\langle\left(\overline{u_{i}}\right)^{<K_{0}}\left(\overline{\mathcal{F}}_{i}\right)^{<K_{0}}\right\rangle$, yields

$$
\begin{aligned}
\left|\left\langle\epsilon_{\ell}^{i n j}-u_{i}^{<K_{0}} \mathcal{F}_{i}^{<K_{0}}\right\rangle\right|= & \left|\left\langle\frac{\bar{\tau}\left(\rho, u_{i}\right)}{\bar{\rho}} \overline{\mathcal{F}}_{i}\right\rangle-\left\langle\bar{\tau}\left(u_{i}^{<K_{0}}, \mathcal{F}_{i}^{<K_{0}}\right)\right\rangle\right| \\
\leq & (\text { const. })\left\|\frac{1}{\rho}\right\|\|\mathcal{F}\|_{4}\left\|\bar{\tau}\left(\rho, u_{i}\right)\right\|_{2} \\
& +\left\|\bar{\tau}\left(u_{i}^{<K_{0}}, \mathcal{F}_{i}^{<K_{0}}\right)\right\|_{1}
\end{aligned}
$$

Similar to bounds (44) and 45 in Proposition 5 , we have

$$
\begin{aligned}
\left\|\bar{\tau}\left(\rho, u_{i}\right)\right\|_{2} & =O\left(\left(\ell K_{0}\right)^{\sigma_{4}^{\rho}+\sigma_{4}^{u}}\right) \\
\left\|\bar{\tau}\left(u_{i}^{<K_{0}}, \mathcal{F}_{i}^{<K_{0}}\right)\right\|_{1} & =O\left(\left(\ell K_{0}\right)^{2}\right),
\end{aligned}
$$

Result (47) follows.

\section{Small-scale kinetic energy budget}

For completeness, we derive the kinetic energy budget at scales $<\ell$ which complements large-scale budget 13. A small-scale kinetic energy may be defined as

$$
\widetilde{k}_{\ell} \equiv \bar{\rho}_{\ell} \frac{\widetilde{\tau}_{\ell}\left(u_{i}, u_{i}\right)}{2}
$$

Integrating $\widetilde{k}_{\ell}$ in space gives $\int d \mathbf{x} \rho|\mathbf{u}|^{2} / 2-\int d \mathbf{x} \bar{\rho}|\widetilde{\mathbf{u}}|^{2} / 2$, which is the total kinetic energy less the energy at large scales. Similar to the large-scale budget 13 , it is straightforward to derive a budget for $\widetilde{k}_{\ell}$, which reads

$$
\partial_{t} \frac{\bar{\rho}_{\ell} \widetilde{\tau}_{\ell}\left(u_{i}, u_{i}\right)}{2}+\nabla \cdot \mathbf{J}_{\ell}^{\text {small }}=\Pi_{\ell}+\Lambda_{\ell}+\bar{\tau}_{\ell}(P, \nabla \cdot \mathbf{u})-D_{\ell}^{\text {small }}+\epsilon_{\ell}^{\text {small }}
$$


The SGS kinetic energy flux terms, $\Pi+\Lambda$ that appear as a sink in the large-scale budget 13) now appear as a source, representing the energy gained by the small-scales from scales larger than $\ell . \mathbf{J}_{\ell}^{\text {small }}$ is spatial transport of $\widetilde{k}, \bar{\tau}_{\ell}(P, \nabla \cdot \mathbf{u})$ is pressure dilatation taking place at scales $<\ell, D_{\ell}^{\text {small }}$ is small-scale viscous dissipation, and $\epsilon_{\ell}^{\text {small }}$ is energy input by external stirring that will vanish on average at scales $\ell \ll K_{0}^{-1}$ for a large-scale $\mathbf{F}^{<K_{0}}$ as we proved in Proposition 5 . These terms are defined as

$$
\begin{aligned}
D_{\ell}^{\text {small }}(\mathbf{x}) & =\overline{\partial_{j} u_{i} \sigma_{i j}}-\partial_{j} \widetilde{u}_{i} \bar{\sigma}_{i j} \\
J_{j}^{\text {small }}(\mathbf{x}) & =\frac{\bar{\rho} \widetilde{\tau}\left(u_{i}, u_{i}\right)}{2} \widetilde{u}_{j}+\frac{1}{2} \bar{\rho} \widetilde{\tau}\left(u_{i}, u_{i}, u_{j}\right)+\bar{\tau}\left(P, u_{j}\right)-\left(\overline{u_{i} \sigma_{i j}}-\widetilde{u}_{i} \bar{\sigma}_{i j}\right) \\
\epsilon_{\ell}^{\text {small }}(\mathbf{x}) & =\bar{\rho} \widetilde{\tau}\left(u_{i}, F_{i}\right),
\end{aligned}
$$

where $\widetilde{\tau}(f, g, h)=\widetilde{f g h}-\widetilde{f} \widetilde{\tau}(g, h)-\widetilde{g} \widetilde{\tau}(f, h)-\widetilde{h} \widetilde{\tau}(f, g)-\widetilde{f} \widetilde{g} \widetilde{h}$ in expression 53 is a Favre-filter analogue of the "generalized central moment" introduced by Germano Germano (1992).

\section{References}

M. Germano, Turbulence - The filtering approach, J. Fluid Mech. 238 (1992) 325-336.

G. L. Eyink, Local energy flux and the refined similarity hypothesis, J. Stat. Phys. 78 (1995) 335-351.

G. L. Eyink, Locality of turbulent cascades, Physica D 207 (2005) 91-116.

A. Favre, Statistical equations of turbulent gases, in: Problems of hydrodynamic and continuum mechanics, SIAM, Philadelphia, pp. 231-266.

S. Kida, S. A. Orszag, Energy and spectral dynamics in forced compressible turbulence, J. Sci. Comput. 5 (1990) 85-125.

P. Chassaing, An alternative formulation of the equations of turbulent motion for a fluid of variable density, J. Mec. Theor. Appl. 4 (1985) 375-389.

H. Aluie, Compressible Turbulence: The Cascade and its Locality, Phys. Rev. Lett. 106 (2011) 174502.

H. Aluie, S. Li, H. Li, Conservative Cascade of Kinetic Energy in Compressible Turbulence, Astro. Phys. J. Lett. 751 (2012) L29.

G. Falkovich, I. Fouxon, Y. Oz, New relations for correlation functions in Navier-Stokes turbulence, J. Fluid Mech. 644 (2010) 465. 
S. Galtier, S. Banerjee, Exact Relation for Correlation Functions in Compressible Isothermal Turbulence, Phys. Rev. Lett. 107 (2011) 134501.

A. Leonard, Energy Cascade in Large-Eddy Simulations of Turbulent Fluid Flows, Adv. Geophys. 18 (1974) A237.

G. L. Eyink, H. Aluie, Localness of energy cascade in hydrodynamic turbulence. I. Smooth coarse graining, Phys. Fluids 21 (2009) 115107.

P. Sagaut, M. Germano, On the filtering paradigm for LES of flows with discontinuities, J. Turbul. 6 (2005) 23 .

E. Garnier, N. Adams, P. Sagaut, Large Eddy Simulation for Compressible Flows, Springer, Netherlands, 2009.

G. L. Eyink, Course notes on turbulence theory, Available online: http://www.ams.jhu.edu/ eyink/OLD/Turbulence_ Spring08/notes.html, 2007.

H. Aluie, Hydrodynamic and Magnetohydrodynamic Turbulence: Invariants, Cascades, and Locality, Ph.D. thesis, The Johns Hopkins University, Baltimore, 2009.

K. R. Sreenivasan, On the scaling of the turbulence energy dissipation rate, Phys. Fluids 27 (1984) 1048-1051.

K. R. Sreenivasan, An update on the energy dissipation rate in isotropic turbulence, Phys. Fluids 10 (1998) 528-529.

B. R. Pearson, T. A. Yousef, N. E. L. Haugen, A. Brandenburg, P. A. Krogstad, Delayed correlation between turbulent energy injection and dissipation, Phys. Rev. E 70 (2004) 056301.

B. Vreman, B. Geurts, H. Kuerten, A priori test of large eddy simulation of the compressible plane mixing layer, J. Eng. Math. 29 (1995) 299-327.

B. Vreman, A priori test of large eddy simulation of the compressible plane mixing layer, Ph.D. thesis, University of Twente, Twente, 1995.

P. Martin, U. Piomelli, G. V. Candler, Subgrid-scale models for compressible large-eddy simulations, Theor. Comp. Fluid Dyn. 13 (2000) 361-376.

T. B. Gatski, J. Bonnet, Compressibility, Turbulence and High Speed Flow, Elsevier, Netherlands, 2009.

H. Tennekes, J. L. Lumley, A First Course in Turbulence., The MIT Press, Cambridge, Massachusetts, 1972. 
R. H. Kraichnan, Kolmogorov's hypotheses and Eulerian turbulence theory, Phys. Fluids 7 (1964) $1723-1734$.

C. G. Speziale, Galilean invariance of subgrid-scale stress models in the large-eddy simulation of turbulence, J. Fluid Mech. 156 (1985) 55-62.

H. Aluie, G. L. Eyink, Localness of energy cascade in hydrodynamic turbulence. II. Sharp spectral filter, Phys. Fluids 21 (2009) 115108.

H. Aluie, G. L. Eyink, Scale Locality of Magnetohydrodynamic Turbulence, Phys. Rev. Lett. 104 (2010) 081101.

H. Aluie, S. Kurien, Joint downscale fluxes of energy and potential enstrophy in rotating stratified Boussinesq flows, EPL (Europhysics Letters) 96 (2011) 44006.

S. H. Starner, R. W. Bilger, LDA measurements in a turbulent diffusion flame with axial pressure, Combust. Sci. Technol. 21 (1980) 259-276.

P. A. Libby, K. N. C. Bray, Countergradient diffusion in premixed turbulent flames, AIAA J. 19 (1981) 205-213.

D. Livescu, J. R. Ristorcelli, Buoyancy-driven variable-density turbulence, J. Fluid Mech. 591 (2007) $43-71$.

D. Livescu, J. R. Ristorcelli, R. A. Gore, S. H. Dean, W. H. Cabot, A. W. Cook, High-Reynolds number Rayleigh-Taylor turbulence, J. Turbul. 10 (2009) 13.

S. K. Lele, Compressibility effects on turbulence, Annual Review of Fluid Mechanics 26 (1994) 211-254.

P. G. Huang, G. N. Coleman, P. Bradshaw, Compressible turbulent channel flows: DNS results and modelling, J. Fluid Mech. 305 (1995) 185-218.

W. Schmidt, C. Federrath, M. Hupp, S. Kern, J. C. Niemeyer, Numerical simulations of compressively driven interstellar turbulence. I. Isothermal gas, Astron. Astroph. 494 (2009) 127-145.

M. R. Petersen, D. Livescu, Forcing for statistically stationary compressible isotropic turbulence, Phys. Fluids 22 (2010) 116101.

C. Federrath, J. Roman-Duval, R. S. Klessen, W. Schmidt, M. Mac Low, Comparing the statistics of interstellar turbulence in simulations and observations. Solenoidal versus compressive turbulence forcing, Astron. Astroph. 512 (2010) A81. 
A. G. Kritsuk, M. L. Norman, P. Padoan, R. Wagner, The Statistics of Supersonic Isothermal Turbulence, Astrophys. J. 665 (2007) 416-431.

R. Wagner, G. Falkovich, A. G. Kritsuk, M. L. Norman, Flux Correlations in Supersonic Isothermal Turbulence, J. Fluid Mech. (accepted for publication). ArXiv:1209.2164.

H. Aluie, Scale locality and the inertial range in compressible turbulence, ArXiv e-prints (2011). ArXiv:1101.0150.

A. M. Obukhov, Structure of the Temperature Field in Turbulent Flows, Izvestiya Akademii Nauk SSSR, Geogr. and Geophys. Ser. 13 (1949) 58-69.

S. Corrsin, On the Spectrum of Isotropic Temperature Fluctuations in an Isotropic Turbulence, Journal of Applied Physics 22 (1951) 469-473.

G. K. Batchelor, Small-scale variation of convected quantities like temperature in turbulent fluid. Part 1. General discussion and the case of small conductivity, Journal of Fluid Mechanics 5 (1959) 113-133.

P. Constantin, E. Weinan, E. S. Titi, Onsager's conjecture on the energy conservation for solutions of Euler's equation, Commun. Math. Phys. 165 (1994) 207-209.

H. Aluie, Scale decomposition in compressible turbulence, arXiv.org (2011). ArXiv:1012.5877v1. 